\title{
MSTO1 mutations cause mtDNA depletion, manifesting as muscular dystrophy with cerebellar involvement
}

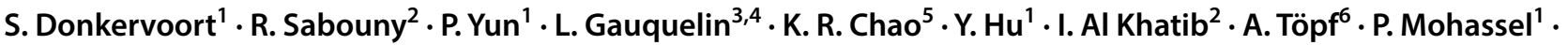 \\ B. B. Cummings ${ }^{5} \cdot$ R. Kaur ${ }^{1}$ - D. Saade ${ }^{1}$ - S. A. Moore ${ }^{7}$. L. B. Waddell ${ }^{8,9} \cdot$ M. A. Farrar ${ }^{10,11}$ - J. K. Goodrich ${ }^{5}$. \\ P. Uapinyoying ${ }^{1,33} \cdot$ S.H. S. Chan ${ }^{12} \cdot$ A. Javed ${ }^{13} \cdot$ M. E. Leach ${ }^{1,14} \cdot$ P. Karachunski ${ }^{15} \cdot$ J. Dalton $^{15} \cdot$ L. Medne $^{16}$. \\ A. Harper ${ }^{17}$. C. Thompson ${ }^{18} \cdot$ I. Thiffault ${ }^{19,20,21}$. S. Specht ${ }^{6} \cdot$ R. E. Lamont ${ }^{22} \cdot$ C. Saunders ${ }^{19,20,21} \cdot$ H. Racher ${ }^{22}$.

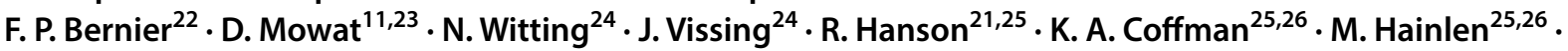 \\ J. S. Parboosingh ${ }^{22} \cdot$ A. Carnevale ${ }^{3}$. G. Yoon ${ }^{3,4} \cdot$ R. E. Schnur ${ }^{27}$. Care4Rare Canada Consortium ${ }^{29} \cdot$ K. M. Boycott ${ }^{28,29}$.

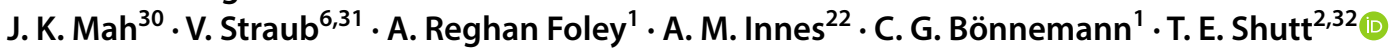

Received: 6 May 2019 / Revised: 25 July 2019 / Accepted: 8 August 2019 / Published online: 29 August 2019

(c) The Author(s) 2019

\begin{abstract}
MSTO1 encodes a cytosolic mitochondrial fusion protein, misato homolog 1 or MSTO1. While the full genotype-phenotype spectrum remains to be explored, pathogenic variants in MSTO1 have recently been reported in a small number of patients presenting with a phenotype of cerebellar ataxia, congenital muscle involvement with histologic findings ranging from myopathic to dystrophic and pigmentary retinopathy. The proposed underlying pathogenic mechanism of MSTO1related disease is suggestive of impaired mitochondrial fusion secondary to a loss of function of MSTO1. Disorders of mitochondrial fusion and fission have been shown to also lead to mitochondrial DNA (mtDNA) depletion, linking them to the mtDNA depletion syndromes, a clinically and genetically diverse class of mitochondrial diseases characterized by a reduction of cellular mtDNA content. However, the consequences of pathogenic variants in MSTO1 on mtDNA maintenance remain poorly understood. We present extensive phenotypic and genetic data from 12 independent families, including 15 new patients harbouring a broad array of bi-allelic MSTO1 pathogenic variants, and we provide functional characterization from seven MSTO1-related disease patient fibroblasts. Bi-allelic loss-of-function variants in MSTO1 manifest clinically with a remarkably consistent phenotype of childhood-onset muscular dystrophy, corticospinal tract dysfunction and earlyonset non-progressive cerebellar atrophy. MSTO1 protein was not detectable in the cultured fibroblasts of all seven patients evaluated, suggesting that pathogenic variants result in a loss of protein expression and/or affect protein stability. Consistent with impaired mitochondrial fusion, mitochondrial networks in fibroblasts were found to be fragmented. Furthermore, all fibroblasts were found to have depletion of mtDNA ranging from 30 to $70 \%$ along with alterations to mtDNA nucleoids. Our data corroborate the role of MSTO1 as a mitochondrial fusion protein and highlight a previously unrecognized link to mtDNA regulation. As impaired mitochondrial fusion is a recognized cause of mtDNA depletion syndromes, this novel link to mtDNA depletion in patient fibroblasts suggests that MSTO1-deficiency should also be considered a mtDNA depletion syndrome. Thus, we provide mechanistic insight into the disease pathogenesis associated with MSTO1 mutations and further define the clinical spectrum and the natural history of MSTO1-related disease.
\end{abstract}

Keywords MSTO1 · Mitochondrial fusion · Cerebellar atrophy $\cdot$ Muscular dystrophy $\cdot$ MtDNA depletion

Electronic supplementary material The online version of this article (https://doi.org/10.1007/s00401-019-02059-z) contains supplementary material, which is available to authorized users.

S. Donkervoort and R. Sabouny are co first authors.

C. G. Bönnemann and T. E. Shutt are co seniors.

Extended author information available on the last page of the article

\section{Introduction}

Mitochondria maintain and express their own genome (mtDNA), typically present in 100-1000 copies per cell and organized into nucleoprotein structures known as nucleoids $[5,18]$. The mtDNA encodes thirteen subunits of the oxidative phosphorylation (OXPHOS) machinery that are essential 
for mitochondrial respiration and ATP production [39]. The relative amount of mtDNA per cell varies in a tissue-specific manner, as an adequate number of mtDNA copies must be maintained to support aerobic respiration and meet cellular energetic demands [24]. As such, reduction in the total amount of mtDNA clinically manifests as severe multi-systemic abnormalities to which energy-demanding organs, such as the brain and muscles, are particularly susceptible.

MtDNA depletion syndromes are a clinically and genetically diverse class of mitochondrial diseases characterized by a reduction of mitochondrial genomes [14,33]. Of the 15 formally defined mtDNA depletion syndromes listed in The Online Mendelian Inheritance in Man database (OMIM) [4], most are caused by pathogenic variants in proteins that are required for mtDNA replication (POLG, C10orf2, MGME1, and TFAM) or those that are necessary to maintain mitochondrial deoxyribonucleoside triphosphates (dNTP) pools (TK2, DGUOK, RRM2B, TYMP, SUCLA2, SUCLG1, AGK, MPV17 and SLC25A) [14, 48]. However, pathogenic variants in proteins that regulate the processes of mitochondrial fusion and fission have also been linked to mtDNA depletion. These include the fusion protein OPA1 (OMIM 616896), as well as MFN2 and DNM1L, which are essential for fusion and fission, respectively [25, 34, 41, 46, 49].

While defects in mtDNA replication or impaired maintenance of mitochondrial dNTP pools are expected to lead to mtDNA depletion, it is less clear how mitochondrial fusion and fission are involved in the regulation of mtDNA. Nonetheless, abnormalities in mtDNA integrity and nucleoid distribution have been demonstrated in several models of defective fusion. Mitochondrial membrane fusion is orchestrated by the activity of large GTPases, including Mitofusin 1 and 2 (MFN1, MFN2) localized to the outer membrane, and Optic Atrophy 1 (OPA1) in the inner mitochondrial membrane. In yeast, cells lacking the Mitofusin homolog Fzo1p suffer from complete loss of mtDNA [21]. Meanwhile, knockout fibroblasts for OPA1, MFN1 and/or MFN2 display fragmented mitochondrial networks with altered nucleoid distribution whereby some fragments are devoid of mitochondrial genomes [10]. Finally, in humans, pathogenic variants in $M F N 2$ and $O P A 1$ cause severe mtDNA depletion and multiple mtDNA deletions [41, 46, 49]. Patients with pathogenic variants in $M F N 2$ or $O P A 1$ that are associated with mtDNA impairments have been reported to present with a phenotype of early-onset ataxia, hypotonia, axonal sensorimotor neuropathy, optic atrophy and hearing loss [2, 3, 22, 41, 46, 49].

Recently, MSTO1 was described as a cytosolic mitochondrial fusion protein, and pathogenic variants in MSTOI have been reported to cause ataxia, muscle weakness, cerebellar atrophy and pigmentary retinopathy $[16,31,36]$. Notably, these phenotypic features have all been previously reported in mtDNA depletion syndromes $[3,14,22]$. To date, five independent studies have described MSTOI variants in 12 patients from seven families $[6,16,31,36]$. While pathogenic MSTOI variants have been linked to impairments in mitochondrial fusion, the consequence of these variants on mtDNA maintenance and its associated clinical spectrum has not been studied extensively.

In this study, we present an extensive phenotypic characterization of 15 new patients from 12 families harbouring a broad array of bi-allelic pathogenic variants in MSTO1 confirming a remarkably consistent and ultimately recognizable clinical phenotype. Additionally, in cultured fibroblasts from seven patients with MSTOI-related disease, we demonstrate loss of MSTO1 protein, significantly fragmented mitochondrial networks, enlarged lysosomal vacuoles, depletion of mtDNA, and alterations to mtDNA nucleoids. Therefore, we demonstrate that bi-allelic loss-of-function variants in the mitochondrial fusion protein MSTO1 impair mtDNA maintenance and fusion and result in mtDNA depletion in fibroblasts, which we establish is associated with a remarkably consistent clinical spectrum of MSTO1-deficiency.

\section{Materials and methods}

\section{Human subjects and samples}

Patients were ascertained through their local neurology and genetics clinics. F2 was identified through GeneMatcher [45]. Written informed consent and age-appropriate assent for study procedures and photographs were obtained by a qualified investigator (protocol 12-N-0095 approved by the National Institute of Neurological Disorders and Stroke, National Institutes of Health, Research Ethics Board of the Hospital for Sick Children, REB \# 1000009004: SCHN Human Ethics Committee 10/CHW/45, University of Calgary Conjoint Health Research Ethics Board). Medical history was obtained and clinical evaluations, including brain MRI and muscle biopsy, were performed as part of the standard diagnostic evaluation. Muscle biopsy slides and available electron microscopy images (EM) were reviewed by investigators. DNA, muscle and skin biopsy samples were obtained according to standard procedures.

\section{Cell culture}

Control and patient fibroblasts were generated from skin biopsies and cultured in MEM media (Gibco, 11095080) containing L-Glutamine and supplemented with $10 \%$ foetal bovine serum (FBS). HeLa cells were grown in DMEM (Gibco, 11965092) supplemented with 10\% FBS. Cells were maintained at $37{ }^{\circ} \mathrm{C}$ and $5 \% \mathrm{CO}_{2}$. HeLa cells were transfected with a mammalian expression vector containing MSTO1-V5 (DNASU, HsCD00440595) using 
Lipofectamine 3000 (Thermo Fisher Scientific, L3000015) according to manufacturer's instructions. Briefly, cells were seeded at $4.5 \times 10^{5}$ in 6 -well plates and allowed to grow overnight. The following day, $2 \mu \mathrm{g}$ of plasmid were used for transfections and cells were left to grow for $24 \mathrm{~h}$ before harvesting.

For genetic rescue experiments, fibroblasts were electroporated with an MSTO1-P2A-mCherry construct using the Amaxa Nucleofector II system (Lonza). The MSTOI sequence was cloned into an AmCyan-P2A-mCherry construct (AmCyan-P2A-mCherry was a gift from Ilpo Huhtaniemi, Addgene plasmid \# 45350; http://n2t.net/addge ne:45350; RRID:Addgene_45350) [40], replacing the mCyan sequence with the MSTOI sequence. An empty vector containing only mCherry was also generated. Fibroblasts grown to 70-80\% confluence were resuspended in OptiMEM media. Next, $1 \times 10^{6}$ cells in $100 \mu \mathrm{L}$ media and $2 \mu \mathrm{g}$ of plasmid DNA were transferred to a sterile $2 \mathrm{~mm}$ electroporation cuvette (VWR 89047-208) and electroporated using the A-024 program. Cells were then plated onto $35 \mathrm{~mm}$ glass bottom dishes or in $100 \mathrm{~mm}$ dishes and incubated for $48 \mathrm{~h}$ prior to further analysis.

\section{Cell sorting}

Following electroporation with either mCherry empty vector or MSTO1-P2A-mCherry, approximately $4 \times 10^{6}$ fibroblast cells were sorted for red fluorescence (and MSTO1 expression) using a $130-\mu \mathrm{m}$ nozzle on a BD FACSAria Fusion (FACSAriaIII) cytometer (BD Biosciences), supported by FACSDiva Version 8.0.1. Genomic DNA was subsequently purified from control and patient mCherry-positive cells as described below.

\section{Western blot}

For Western analyses, $3 \times 10^{5}$ cells were seeded in $100 \mathrm{~mm}$ plates, allowed to grow for 2-3 days, harvested by trypsin digestion, washed with $1 \mathrm{X}$ phosphate-buffered saline (PBS) and lysed with RIPA buffer containing protease inhibitors. Total cell lysates $(50 \mu \mathrm{g})$ were resolved on SDS-PAGE gels and transferred onto PVDF membranes. Blots were subsequently probed with the following antibodies (1:1000 dilution unless otherwise indicated): anti-MSTO1 (Genetex, GTX105110) (1:500), anti-V5 (Millipore, AB3792), anti-Mitofusin1 (Cell Signalling, 14739), anti-Mitofusin2 (Abnova, H00009927-M03), anti-Opa1 (BD Bioscience, 612606), anti-Actin (Sigma A5316), anti-HSP60 (Cell Signalling, 12165), VDAC1 (Abcam, ab14734) and the appropriate horseradish peroxidase (HRP)-conjugated secondary antibodies (1:3000). Blots were incubated with Clarity ECL substrate (Biorad, 1705061) and imaged on an Amersham Imager AI600.

\section{mtDNA copy number analysis}

Total genomic DNA (gDNA) (nuclear and mitochondrial DNA) was extracted from control and patient fibroblasts using the PureLink Genomic DNA Mini Kit (Thermo Fisher Scientific, K182001) according to manufacturer's instructions. Relative mtDNA copy number was analysed by realtime quantitative PCR (qPCR) using the QuantStudio 6 Flex Real-Time PCR system (Thermo Fisher Scientific). Primer sequences specific to mtDNA, nuclear-encoded housekeeping gene $18 \mathrm{~S}$ and thermocycling conditions were performed as described previously [13].

QPCR reactions were prepared to a total of $20 \mathrm{uL}$ per reaction containing $10 \mathrm{uL}$ PowerUp SYBR Green Master Mix (Thermo Fisher Scientific, A25742), 100 ng gDNA and $500 \mathrm{nM}$ forward and $500 \mathrm{nM}$ reverse primers. MtDNA copy number relative to $18 \mathrm{~S}$ was analysed using the delta delta $\mathrm{Ct}$ method and represented as percent control [32]. Relative mtDNA copy number was presented as mean \pm SD from at least three independent biological replicates and unpaired, 2-tailed Student's $t$ tests were used to determine statistical significance.

\section{Immunofluorescence staining}

Fibroblasts were seeded on $12 \mathrm{~mm}$ glass coverslips (no. 1.5) at $2 \times 10^{4}$ cells and allowed to grow for $24 \mathrm{~h}$. Cells were subsequently fixed and stained with primary antibodies against TOMM20 (Santa Cruz Biotechnology, FL-145) and LAMP1 (Santa Cruz Biotechnology, 18821) in addition to appropriate Alexafluor-conjugated secondary antibodies (Thermo Fisher Scientific) at 1:1000 as done previously [42].

\section{Live cell imaging}

In order to visualize mitochondrial DNA nucleoids, fibroblasts were stained with PicoGreen (Thermo Fisher Scientific, P7581) as described previously [7]. Briefly, cells were seeded on glass bottom dishes (Mattek, P35G-1.5-14-C) at $8 \times 10^{4}$ and incubated overnight. Approximately $1 \mathrm{~h}$ prior to imaging, cells were stained with PicoGreen at $3 \mu \mathrm{L} /$ $\mathrm{mL}$ for $30-45 \mathrm{~min}$ at $37{ }^{\circ} \mathrm{C}$. MitoTracker Red dye (50 nM) (Thermo Fisher Scientific, M7512) or MitoTracker Deep Red dye (50 nM) (ThermoFischer Scientific, M22426) was also added to the media to visualize mitochondrial networks. The media containing dyes was aspirated, cells were washed four times in pre-warmed 1XPBS, and fresh pre-warmed media was added to the cells.

\section{Microscopy}

Images from both fixed and live samples were acquired on an Olympus spinning disc confocal system (Olympus SD OSR) 
(UAPON 100XOTIRF/1.49 oil objective) operated by Metamorph software. A cell Vivo incubation module was used to maintain cells at $37{ }^{\circ} \mathrm{C}$ and $5 \% \mathrm{CO}_{2}$ during live cell imaging.

\section{Image analysis}

\section{Mitochondrial networks}

Mitochondrial morphology was qualitatively analysed by classifying networks into one of four categories, as previously described [16]. For each fibroblast line, at least 50 cells were scored, and the analyses were performed on three independent replicates. Results represent mean $\pm \mathrm{SD}$, and $P$ values were based on unpaired, 2-tailed Student's $t$ tests.

\section{MtDNA nucleoids}

Mitochondrial DNA nucleoid size and number were analysed in 35 fibroblast cells from each patient line and control using the particle analysis tool in ImageJ FIJI [43]. First, images were acquired using the same acquisition parameters, scaled and binarized. For each cell, a region of interest (ROI) encompassing the entire mitochondrial network was selected. Then, in binarized mtDNA nucleoid images, the particle analysis tool was used to measure surface area and total nucleoid counts within the selected ROI. Nuclear signal was excluded from the analysis. Nucleoid sizes are presented as the average size of all nucleoids per cell in a violin plot, highlighting the distribution of quantified mtDNA nucleoid sizes. The non-parametric Kolmogorov-Smirnov (K-S) test was used to determine statistical significance regarding the distribution of nucleoid sizes. The relative frequencies of nucleoid sizes were assessed in each quantified cell and the percentage of large nucleoids $(>0.2$ $\mu \mathrm{m}^{2}$ ) in control and patient fibroblasts was plotted. Nucleoid counts represent mean $\pm \mathrm{SD}$, and $P$ values were based on unpaired, 2-tailed Student's $t$ tests.

\section{Lysosomes}

The presence of enlarged lysosomal aggregates was quantified from confocal images of patient and control cells by scoring the number of cells with normal vs. enlarged lysosomes. At least 50 cells were analysed per fibroblast line from two independent replicates. Results represent mean $\pm \mathrm{SD}$, and $P$ values were obtained from unpaired, 2-tailed Student's $t$ tests.

\section{Results}

\section{Clinical characteristics}

The clinical presentation of the 15 patients, which includes nine females and six males, is summarized in Table 1, with ages ranging from 6 to 52 years at the time of most recent examination. There were two families with more than one affected relative: Family 1 (F1) consists of three affected sisters [P1, P2, P3; p.(Asp236His); p.(Arg279His)], and Family 2 (F2) consists of two affected siblings [P4, P5; p.(Gly420ValfsX2); p.(Arg256Gln)] (Fig. 2d). Family history was non-contributory in the remaining patients of the cohort.

Patients typically presented with hypotonia and delayed motor milestones with first symptoms recognized between birth and three years of age. All but five patients presented with delayed motor milestones and then subsequently achieved independent ambulation with gaits characterized as waddling-like and wide-based across the entire cohort. All 15 patients reported either relatively slow progression or no progression of their muscle weakness. At the time of the last examination, all were found to have predominantly proximal weakness. Cerebellar symptoms manifested as dysmetria in 11, gait ataxia in nine and abnormal speech in 12, which included a history of speech delay in five and dysarthria in eight patients, respectively. Corticospinal tract manifestations including increased tone, the presence of a spastic catch, clonus and/or increased deep tendon reflexes were observed in eight patients. A history of learning difficulties was reported in nine patients while none of the patients were found to have major cognitive involvement. None of the patients had a history of seizures, cataracts, hearing or cardiac involvement.

Electrophysiological studies were available for four patients. Three [P4: p.(Gly420ValfsX2); p.(Arg256Gln), P5: p.(Gly420ValfsX2); p.(Arg256Gln), P10; p.(Asp236Gly); p.(Arg279His)] of the four had evidence of myotonia on EMG, and complex repetitive discharges were present in P8 [p.(Phe217Leu); p.(Asp236His)]. Lower extremity muscle MRI imaging was available for four patients and revealed a spectrum of involvement ranging from severe involvement of the muscles of the upper leg with apparent fatty replacement of all muscles except for the semimembranosus and the biceps femoris (P6; p.(Asp236His); p.(Phe217Leu)) to mild fatty infiltration of upper and lower leg muscles [P10; p.(Asp236Gly); p.(Arg279His)] (Table 1; Fig. 1a). Pulmonary function testing was performed in nine patients and revealed reduced forced vital capacity (FVC) measurements, ranging from 60 to $79 \%$ predicted. Serum creatine kinase (CK) levels were significantly increased in all patients, except for P15, ranging from 300 to $5000 \mathrm{U} / \mathrm{L}$. Echocardiogram was normal in the 11 patients who underwent this screening evaluation.

Overall, we recorded a remarkably consistent clinical phenotype of primary motor developmental delay, fairly stable mostly proximal muscle weakness caused by a muscular dystrophy, mild cerebellar findings of dysmetria, ataxia and dysarthria based on a stable congenital cerebellar atrophy, 


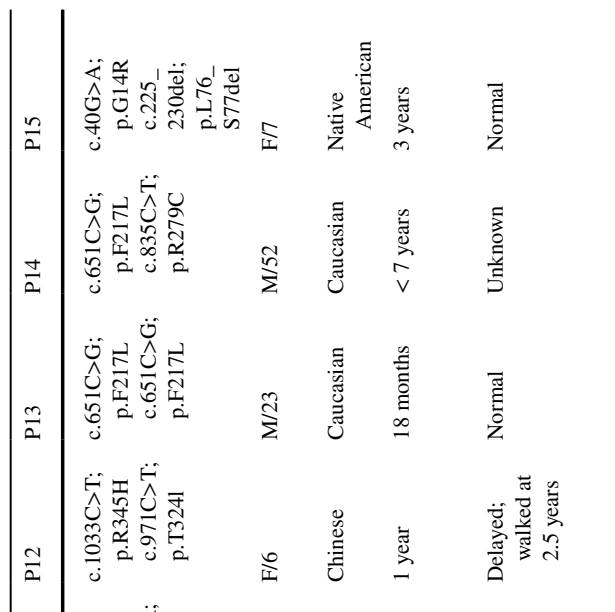

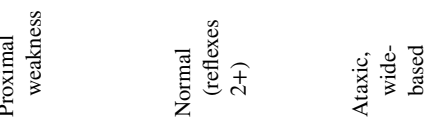

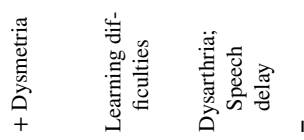

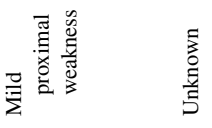
总

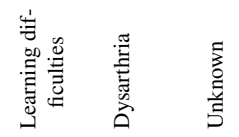

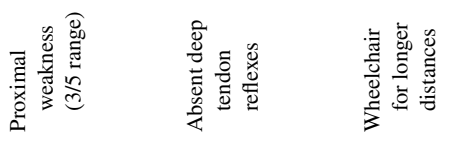

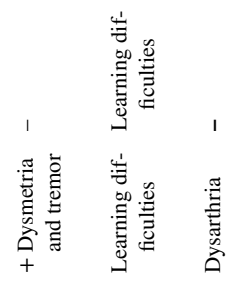

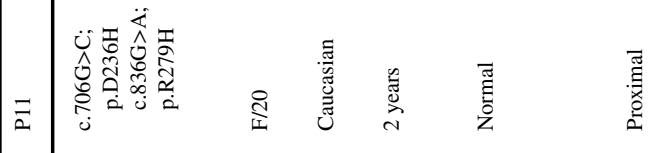

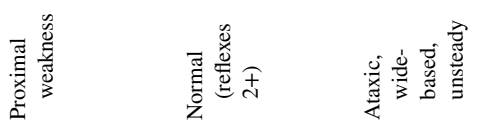

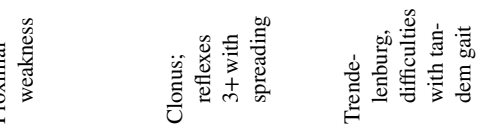

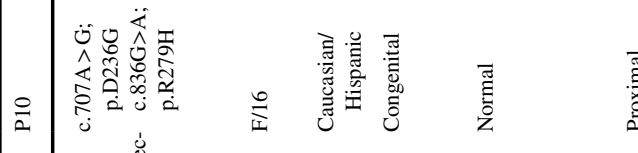

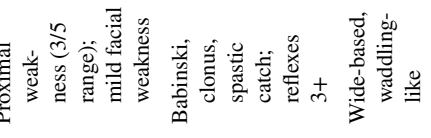

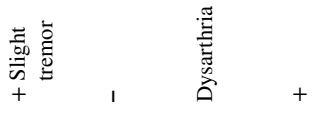

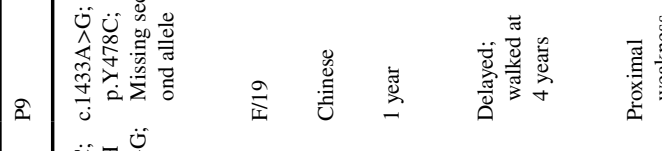

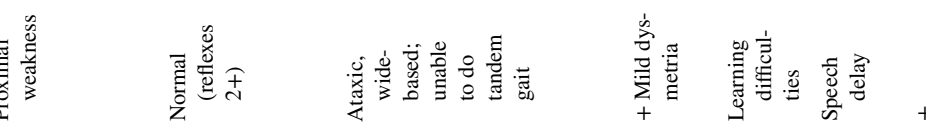

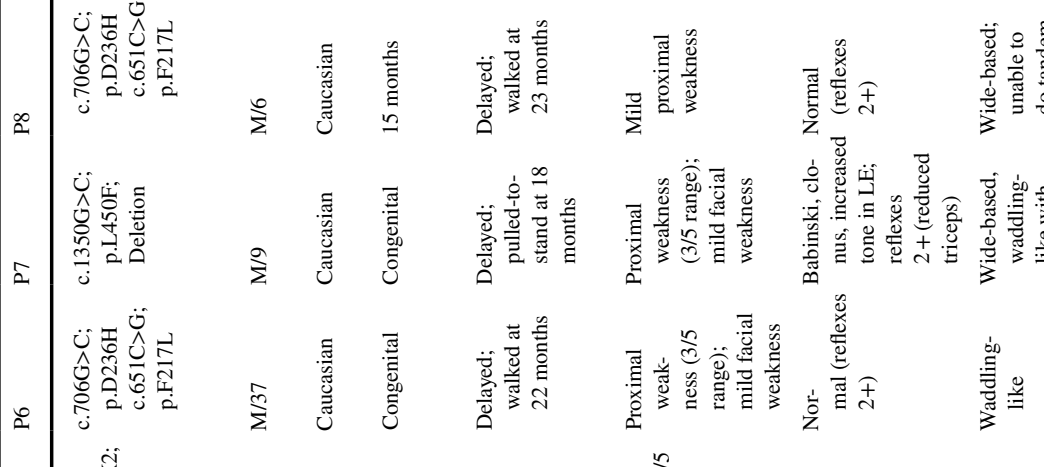

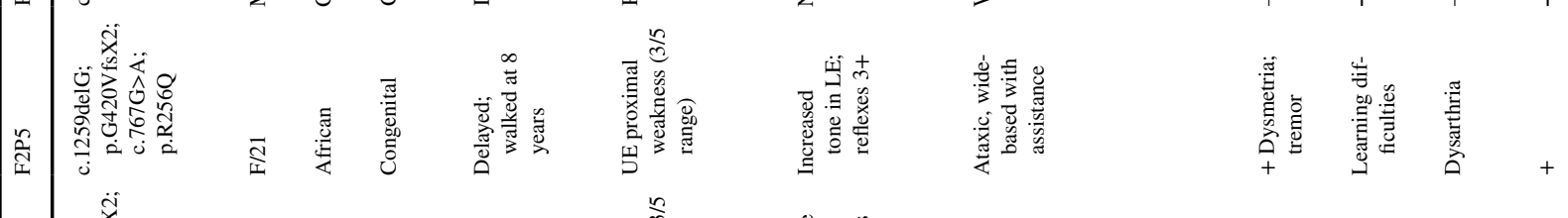

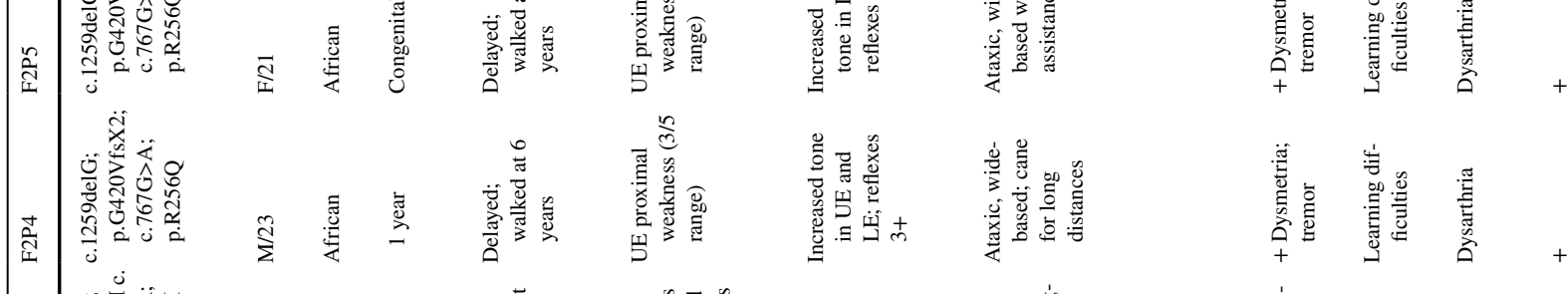

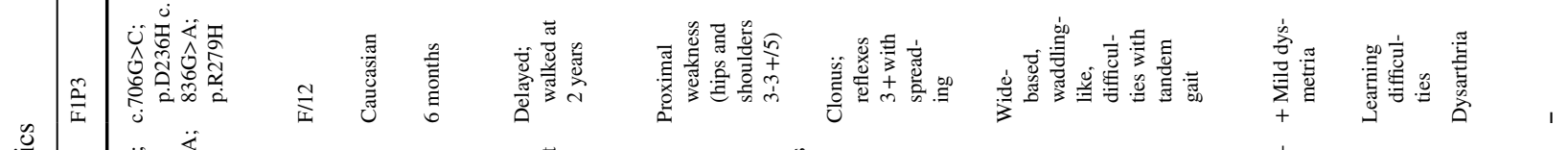

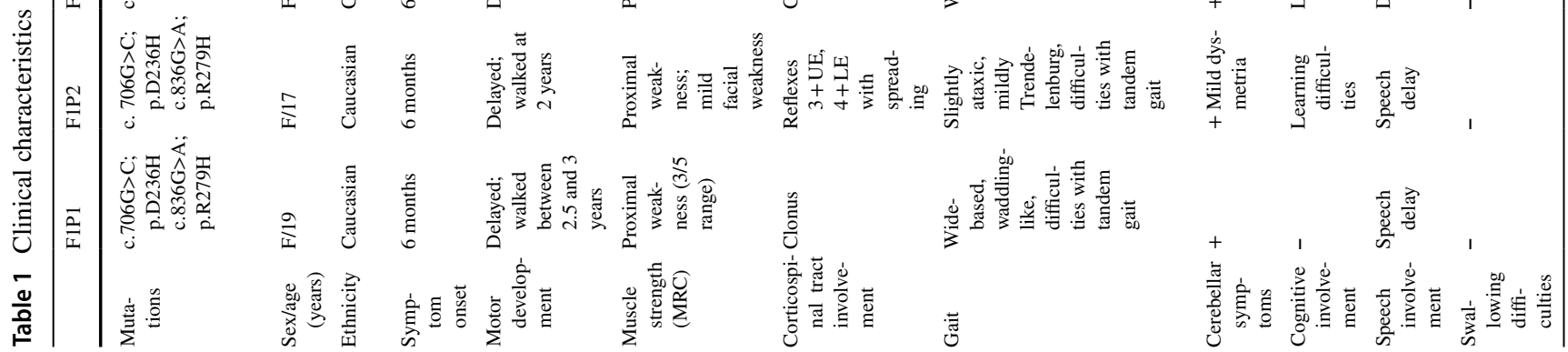




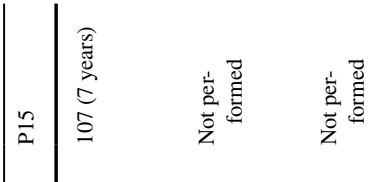

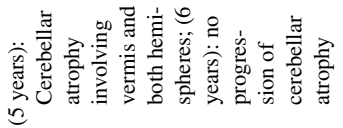

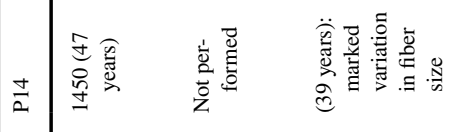
竞营

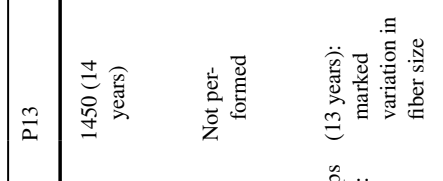
离营

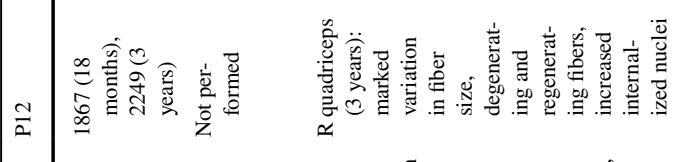

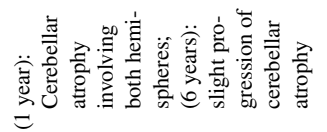

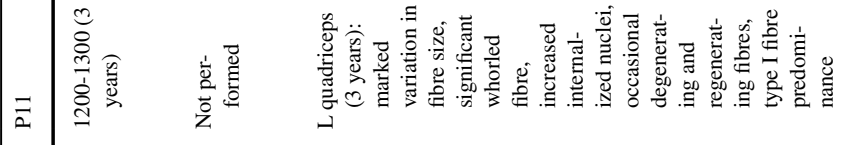

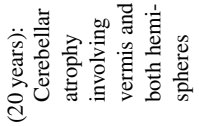

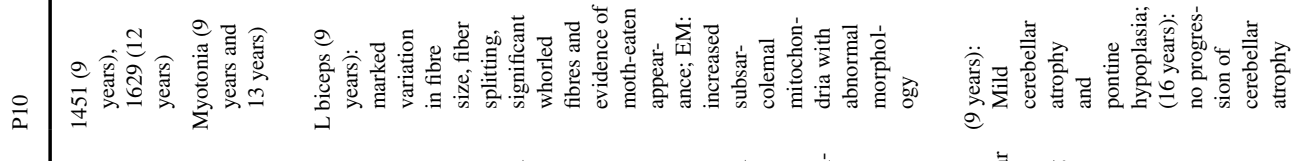

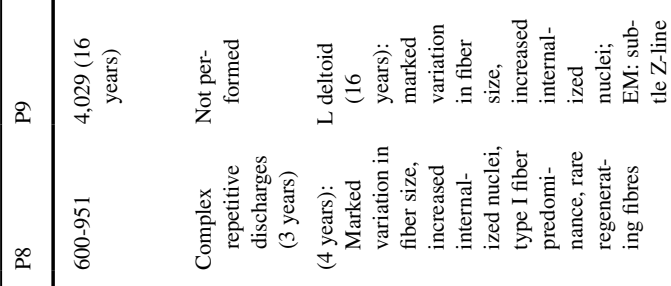

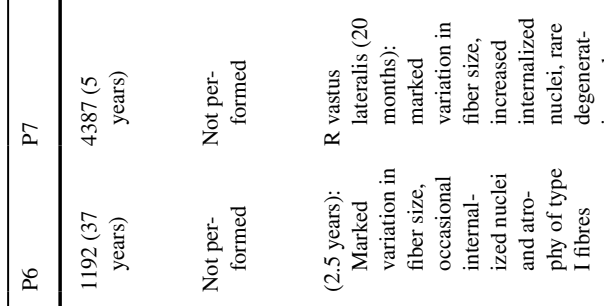

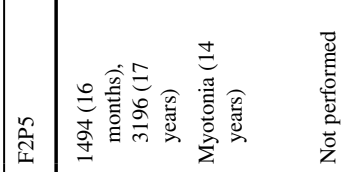

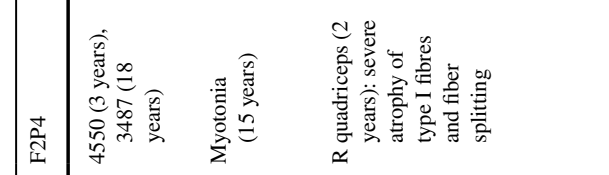

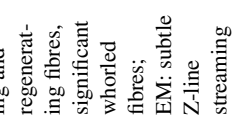

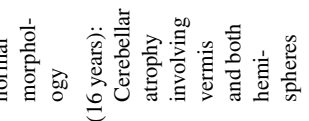

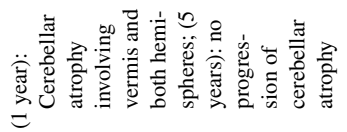

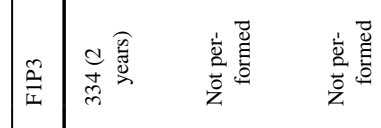

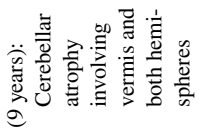
竞

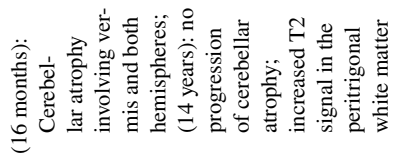

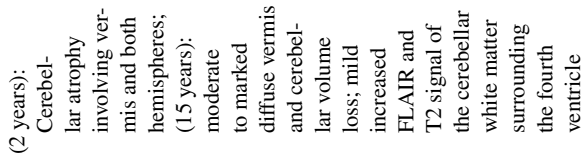

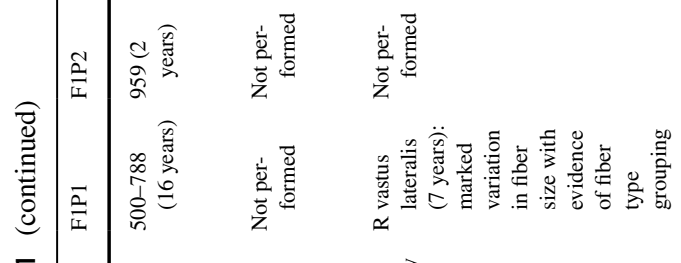

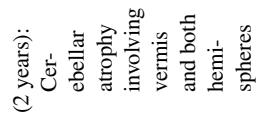

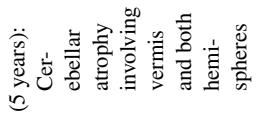

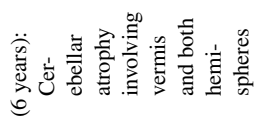

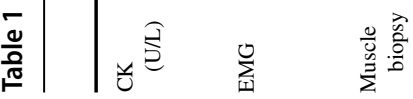
高竞 


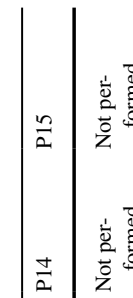

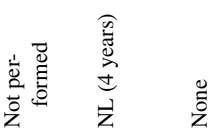

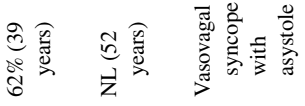

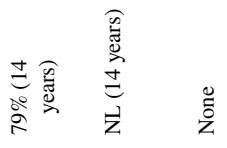

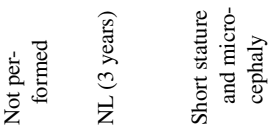
च

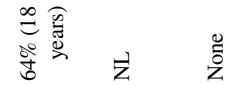

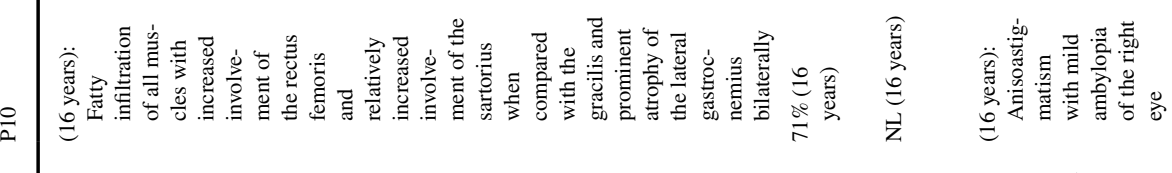
๙

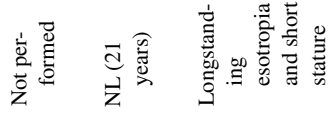

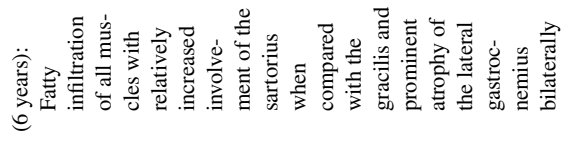

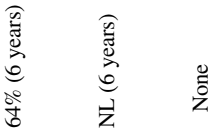
ڤ|

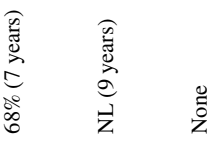

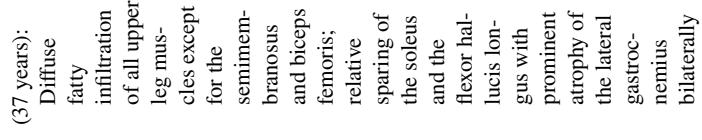

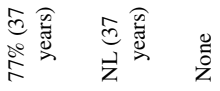

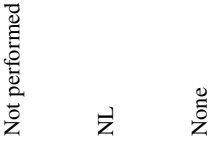
总

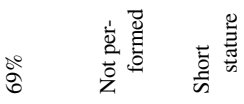
犬े

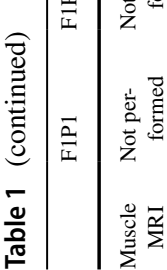

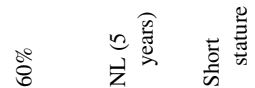

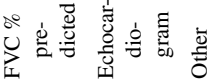




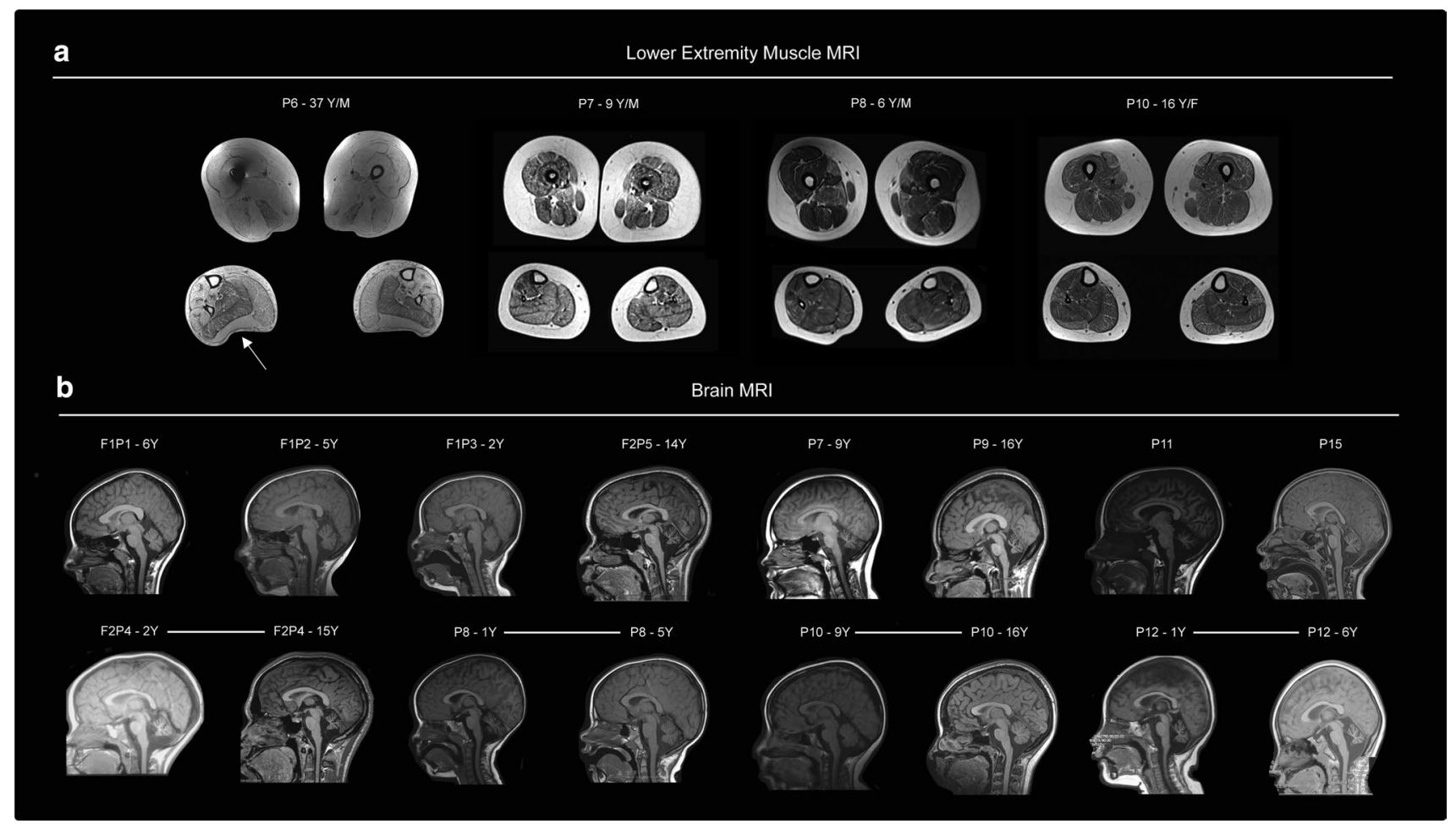

Fig. 1 Muscle and brain imaging. a Lower extremity muscle MRI of patients P6 [p.(Asp236His); p.(Phe217Leu)], P7 [p.(Leu450Phe); deletion], P8 [p.(Phe217Leu); p.(Asp236His)] and P10 [p.(Asp236Gly); p.(Arg279His)] at ages 37 years, 9 years, 6 years and 16 years, respectively. Abnormal signal intensity of muscles such as the posterior gastrocnemius muscle in patient P6 (white arrow), reflects muscle breakdown and replacement with adipose tissue. b Brain MRI completed in 12 patients consistently demonstrates mod-

mild pyramidal signs and evidence for some degree of speech delay and learning disability in some. Meanwhile, major cognitive involvement, seizures, retinopathy, optic atrophy or hearing loss were not seen.

\section{Neuroimaging characteristics}

Brain MR imaging was available for 12 patients and consistently showed moderate to severe cerebellar atrophy/ hypoplasia involving the vermis and both hemispheres in all 12 (Fig. 1b). Four of the brain MRIs had been performed in patients before age 2 years, which revealed significant decrease in cerebellar volume. A lack of progression of cerebellar volume loss was confirmed through repeat imaging available in four patients. Patient 12 [p.(Arg345His); p.(Thr324Ile)] showed mild progression of cerebellar volume loss between ages one and 6 years. erate-to-severe cerebellar volume loss or hypoplasia involving the vermis and both hemispheres. Repeat MRI images were available in patients P4 [p.(Gly420ValfsX2); p.(Arg256Gln)], P8 [p.(Phe217Leu); p.(Asp236His)], P10 [p.(Asp236Gly); p.(Arg279His)] and P12 [p.(Arg345His); p.(Thr324Ile)] demonstrate mild [P12] to no progression [P4, P8 and P10] of cerebellar volume loss over time (second row)

\section{Muscle histopathology and electron microscopy}

Muscle biopsies were performed in ten patients and were consistent with a dystrophic process with evidence of variation in fibre type size, a mild degree of necrosis and regeneration, internalized nuclei and whorled fibres (Fig. 2a). Electron microscopy analysis was performed in three patients. Aggregates of subsarcolemmal mitochondria were noted in patients P9 [p.(Tyr478Cys); missing] and P10 [p.(Asp236Gly); p.(Arg279His)]. There was also evidence of non-specific mitochondrial morphologic abnormalities (variations in mitochondrial shape and size) seen in patients P9 and P10 (Fig. 2b).

\section{Molecular results}

Using whole exome sequencing (WES), we identified apparent homozygous or compound heterozygous variants in MSTO1 (NM_018116.3) in 15 patients from 12 independent families consistent with the bi-allelic recessive mode of inheritance (Fig. 2c). Parental DNA for segregation testing 
a
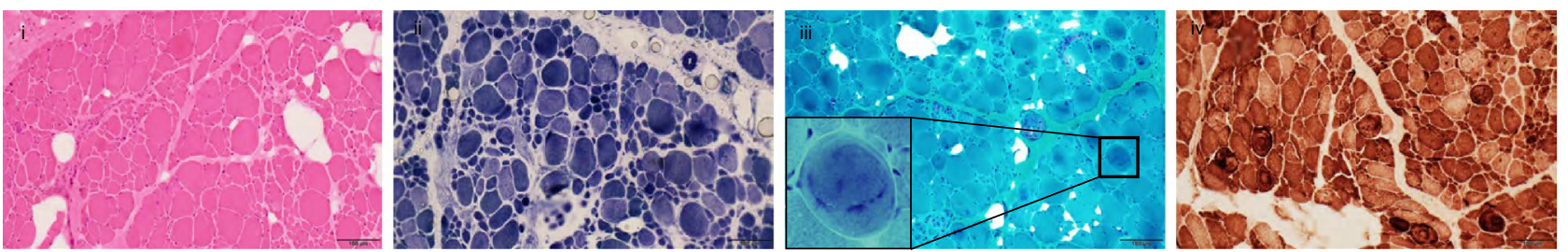

b
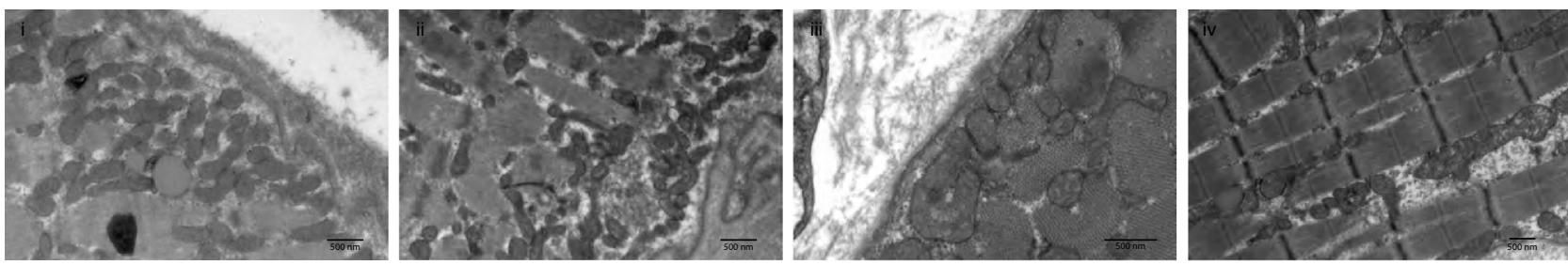

C

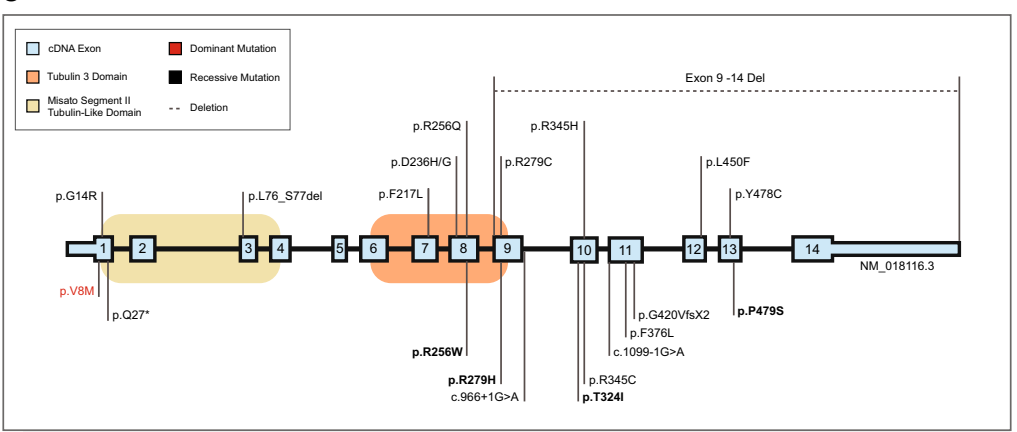

d

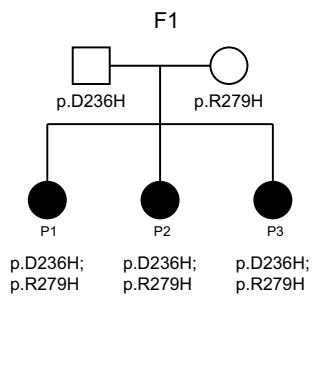

F2

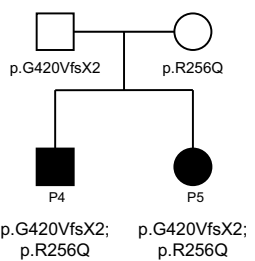

Fig. 2 Muscle biopsy, MSTO1 pathogenic variants and pedigrees. a Histology findings from the vastus lateralis muscle biopsy of P7 [p.(Leu450Phe); deletion] at age 20 months include internalized nuclei on hematoxylin and eosin (H\&E) staining (white arrow) (i) and variation in fiber size on nicotinamide dinucleotide (NADH) staining (ii) and whorled fibres evident on Gömöri trichrome (inset) (iii) and COX staining (white arrow) (iv). b Muscle biopsy electron microscopy (EM) findings are notable for aggregates of subsarcolemmal mitochondria in both P9 [p.(Tyr478Cys); missing)] (i and ii) and P10 [p.(Asp236Gly); p.(Arg279His)] (iii and iv) and non-specific mitochondrial morphologic abnormalities (variations in mitochondrial shape and size) in P10. c Schematic of new and reported human
MSTO1 pathogenic variants. Shown in numbered light blue squares are cDNA exons (RefSeq isoform NM_018116.3 of MSTO1). Corresponding known protein domains are shown in orange (tubulin 3 domain) and beige (Misato segment II tubulin-like domain). Variants written in black text are recessive; the single mutation in red has been previously reported to cause dominantly inherited MSTO1-related disease. The top half of the figure depicts novel variants reported in this publication; the bottom half of the figure depicts variants which have been previously reported. Bolded variants depict previously reported mutations that were also present in our cohort. The dotted line depicts a large deletion (exons 9-14). d Pedigree of two families consistent with recessive inheritance of MSTO1 pathogenic variants was not available for P13, who was found to be apparently homozygous for the common p.(Phe217Leu) variant. Six of these missense variants have not yet been reported. The p.(Gly420ValfsX2) frameshift variant was recently reported [31]. There were three apparent recurring mutation hotspots (p.Asp236, p.Arg279, p.Phe217), which were identified as five, four and four independent alleles, respectively. The p.(Arg279His) variant had been previously reported in heterozygosity with a second pathogenic allele in three families [23, 31].

Trio WES identified an apparently homozygous p.(Leu450Phe) MSTO1 pathogenic variant in P7. Targeted sequencing confirmed that this variant was paternally inherited, while the mother was found to be negative for the variant. Subsequent testing using exon-level oligo $\mathrm{CGH}$ array identified a presumed maternally inherited deletion in P7 encompassing at least exons 9-14 of the MSTO1 gene and extending to include both the $Y Y 1 A P 1$ as well as the DAP3 genes (Genomic Coordinates: $\operatorname{arr}[\mathrm{GRCh} 37]$ 1q22(155582110_155708204)x1). Recessive pathogenic variants in YY1APl have been reported in association with Grange syndrome (OMIM 607860). DAP3 has not yet been associated with human disease.

Trio WES for P9 identified a heterozygous p.(Tyr478Cys) MSTO1 variant inherited from a clinically unaffected father. Whole genome sequencing (WGS) in 
P9 confirmed this heterozygous missense variant but did not identify a second MSTO1 allele in compound heterozygosity. Exon-level oligo CGH testing for P9 was normal. RNA sequencing (RNA-seq) analysis to identify any possible transcriptional aberrations in MSTO1 for P9 was inconclusive, and no splice aberrations were identified in P9. Attempts to evaluate allele balance at the hg 19: chr1:155583319 variant via RNA-seq were unsuccessful due to insufficient coverage in the region, likely due to the presence of a highly homologous pseudogene.

Parental segregation testing for all MSTO1 variants was consistent with bi-allelic recessive inheritance, except for P9 (p.(Tyr478Cys); missing) in whom the presumed maternally inherited allele has not yet been identified, and for P13 (p.(Phe217Leu); p.(Phe217Leu)) in whom parental DNA was not available. Variants identified were predicted to be damaging and either absent or extremely rare (allele frequency below 0.00005) in Genome Aggregation Database (GnomAD) and Exome Aggregation Consortium (ExAC) except for the p.(Arg279His) variant. This particular variant was listed with an allele frequency of 0.00019 in ExAC and 0.00026 in GnomAD with one reported homozygous individual [29]. MSTO1 variants are scattered throughout the gene and do not seem to cluster in a specific MSTO1 domain (Fig. 2c).

\section{Characterization of patient fibroblasts}

In order to further investigate the effect of MSTO1 mutations, we examined the expression of MSTO1 protein by immunoblotting. As the MSTO1 antibody we used detects multiple bands in fibroblast cells, we confirmed the size of the correct band, which migrates at the predicted size of $62 \mathrm{kDa}$, corresponding to MSTO1 protein in HeLa cells overexpressing a V5-epitope-tagged-MSTO1 or an empty vector (Fig. 3a). Our data show that the MSTO1 protein is undetectable in all patient fibroblasts, $(n=7)$ suggesting that pathogenic variants affect protein expression and/or stability (Fig. 3a). Although this observation was expected in cells with large deletions encompassing the MSTO1 gene [e.g. P7; p.(Leu450Phe); deletion)], it is intriguing that all patient fibroblasts containing combinations of missense mutations exhibit a similar cellular phenotype. It is also worth noting that $M S T O 1$ variants do not have gross effects on the expression of mitochondrial fusion proteins (MFN1/2 and OPA1) (Fig. 3b).

Given the established role of MSTO1 as a mitochondrial fusion regulator $[16,36]$, we examined mitochondrial morphology in seven MSTO1 patient fibroblasts. Consistent with previous reports, visualized mitochondrial networks in patient fibroblasts were fragmented compared to control fibroblasts (Fig. 4). In addition, we examined lysosomal structures in MSTO1 patient fibroblast lines by

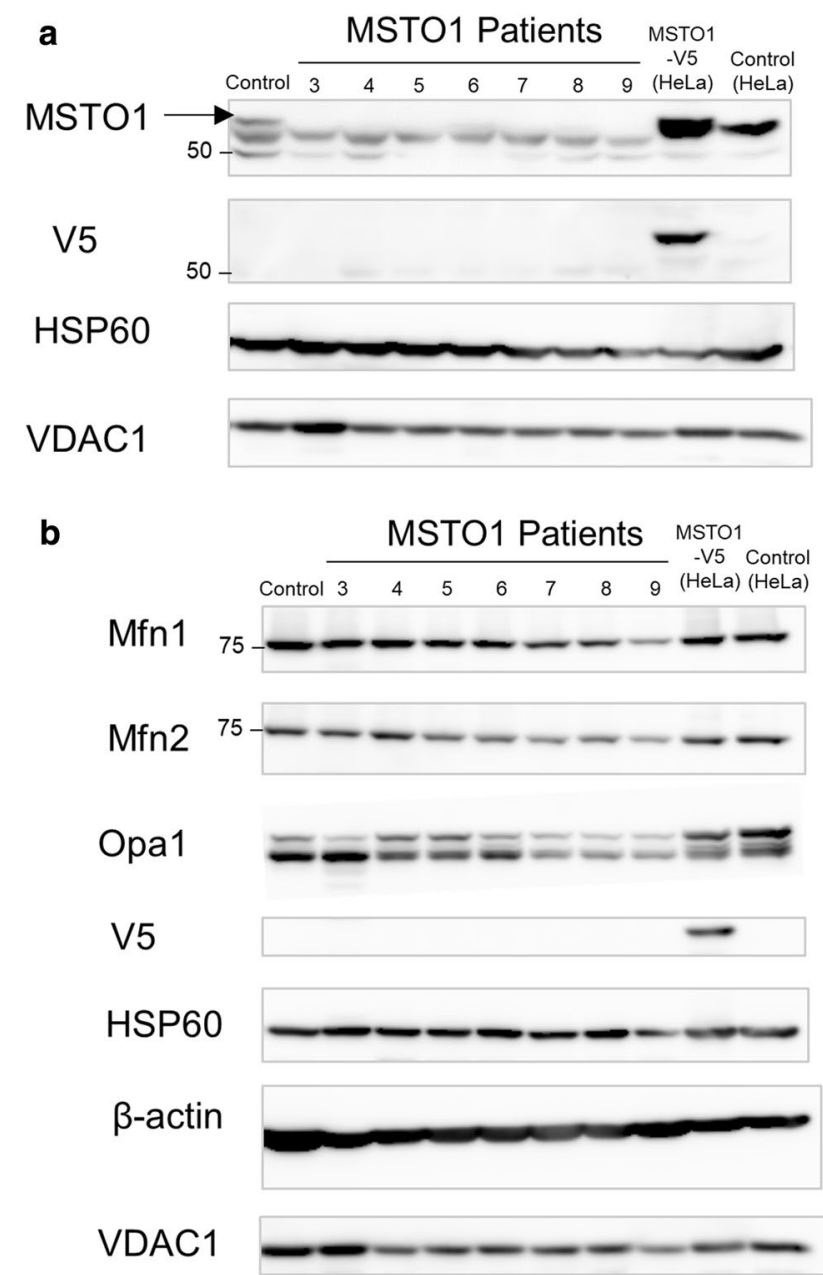

Fig. 3 Pathogenic variants lead to MSTO1 protein instability. a Western blot analysis of total cell lysates from control and patient fibroblast. As a control, total cell lysates from HeLa cells overexpressing MSTO1-V5 or empty vector were also included. Blots were probed with antibodies against endogenous MSTO1, VDAC1, HSP60 and V5. Black arrow corresponds to endogenous MSTO1 protein further verified in HeLa cell lysates; meanwhile, bands underneath are nonspecific. b Western blot analysis of cell lysates as in a. Blots were probed against fusion proteins (Mfn1, Mfn2 and Opa1) and loading controls

immunofluorescence, as mitochondrial dysfunction can also cause alterations to lysosomes [12]. Compared to unaffected control cells, we observed markedly enlarged lysosomal vacuoles across all MSTO1 patient lines (Fig. 5), an observation that has not been previously reported in the context of MSTO1 dysfunction [16, 35].

As pathogenic variants in mitochondrial fusion proteins MFN2 and OPA1 have been shown to cause mtDNA depletion [3, 49], we analysed mitochondrial genomes in patient cells. We observed a significant reduction in mtDNA copy number across all fibroblast lines, ranging from 30 to $70 \%$ depletion (Fig. 6a). MtDNA nucleoids are nucleoprotein assemblies involved in the organization and segregation 
Fig. 4 Characteristics of MSTO1 patient fibroblasts. a Representative confocal microscopy images of control and patient cells. Mitochondrial networks in MSTO1 patient cells are more fragmented and contain fewer but larger mtDNA nucleoids compared to the control cells. Live cells were stained with MitoTracker Red (red, mitochondria) and PicoGreen (green, nuclear and mitochondrial DNA). b Quantification of mitochondrial morphology from control and patient cells performed from three independent replicates. Statistical analysis was performed on the number of cells with partly fragmented mitochondrial morphology in control versus patient cells; Student $T$ test, $* p<0.05$, $* * p<0.001$
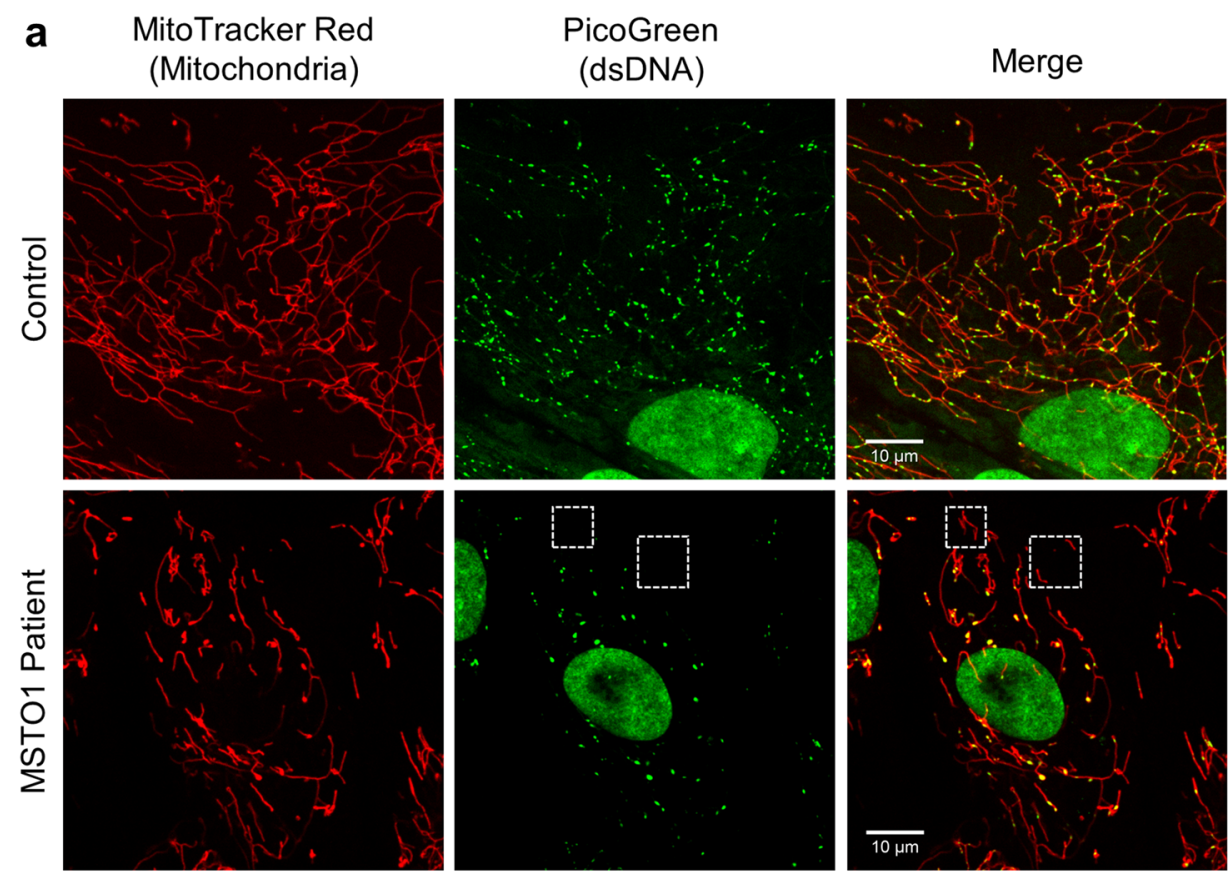

b

Mitochondrial Morphology

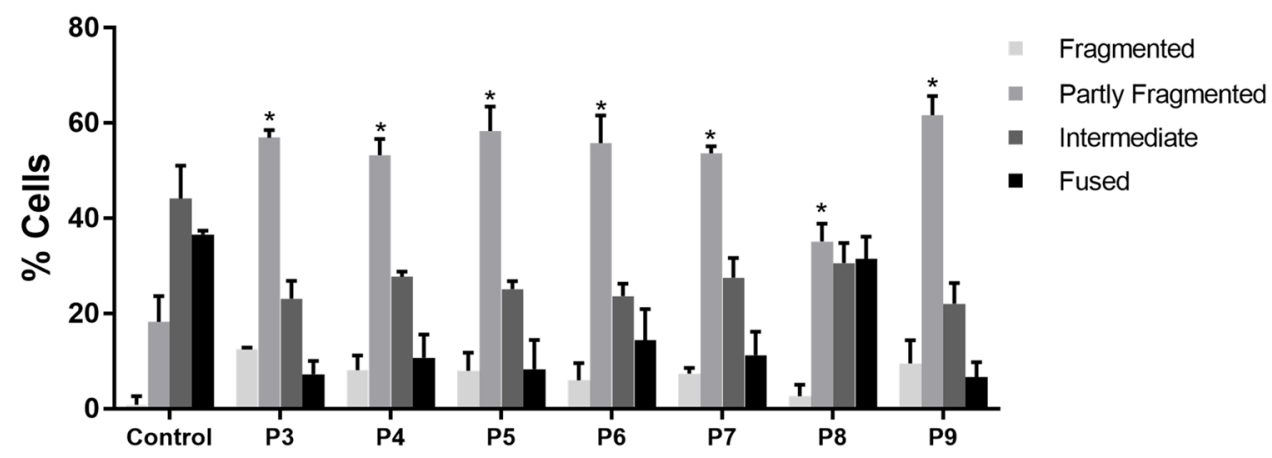

of mtDNA. While examining the size and distribution of mtDNA nucleoids within the mitochondrial network in MSTOI patient fibroblasts, we found that the patient fibroblasts contained fewer nucleoids, which were larger in size compared to control lines (Fig. 6b, c). Notably, several mitochondrial fragments were devoid of mitochondrial genomes in patient cells (Fig. 4a), a phenotype previously reported in cells lacking fusion regulation [10]. Together, these observations demonstrate significant alterations of the mitochondrial genome in all patient fibroblast lines evaluated. Unfortunately, muscle tissue was not available for further mtDNA content studies.

The similarity and consistency of the cellular phenotypes described across all seven MSTO1 patient fibroblast lines strongly support the notion that loss of MSTO1 function is the underlying cause responsible for these observations. In order to further confirm that the cellular phenotypes were in fact due to the loss of MSTO1, we transiently expressed wild-type MSTO1 in two of the patient cell lines (P4 and P7) (Fig. 7a, b). Similar to previous reports [16, 35], we found that expression of wild-type MSTO1 restored mitochondrial morphology after $48 \mathrm{~h}$ (Fig. 7c). Notably, we also observed more fused mitochondrial networks in control cells overexpressing MSTO1, further validating the role of MSTO1 in promoting fusion. In addition, we also see that lysosome abnormalities are restored (Fig. 7d). While we observed a significant rescue in MSTO1 fibroblasts with regard to mtDNA nucleoid size (Fig. 7e), the number of mtDNA nucleoids did not change in MSTO1 fibroblasts (Fig. 7f). A potential confounding factor is that the transfection protocol itself causes a decrease in mtDNA nucleoid counts, which could be masking a rescue. However, mtDNA copy number was also not rescued after only $48 \mathrm{~h}$ (Fig. $7 \mathrm{~g}$ ). This incomplete rescue of mtDNA nucleoid abundance and copy number likely reflects the fact that it may take longer than $48 \mathrm{~h}$ for the mtDNA copy number to be re-established. 
Fig. 5 Enlarged lysosomal vacuoles in MSTO1 patient fibroblasts. a Representative confocal images of control and patient cells fixed and stained with antibodies against TOMM20 (red, mitochondria) and LAMP1 (green, lysosomes). Compared to an unaffected control, patient cells contain distinct lysosomal clusters. $\mathbf{b}$ Quantification of cells containing enlarged lysosomes in control and patient fibroblasts performed from two independent replicates. Statistical analysis was performed; Student $T$ test, ${ }^{*} p<0.05$
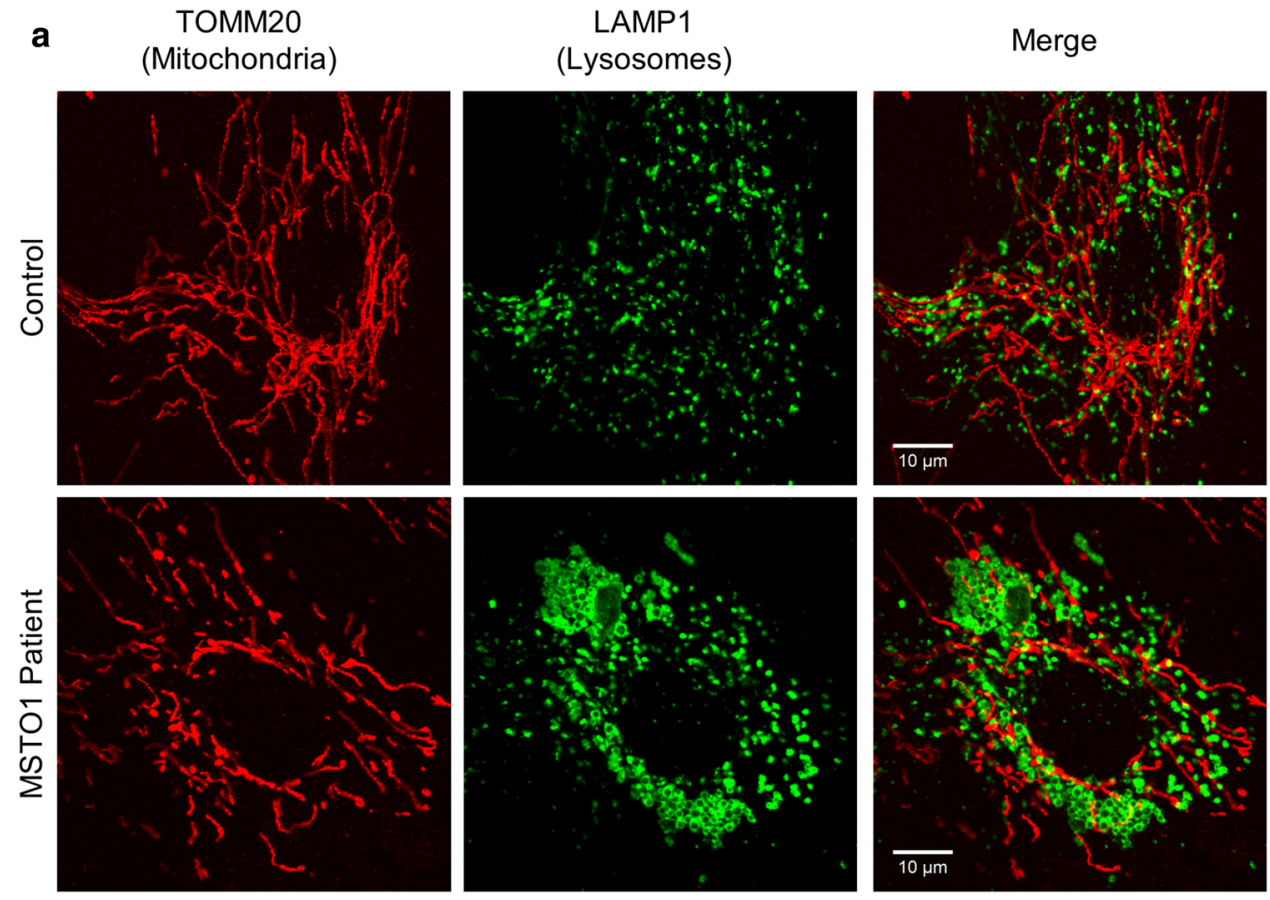

b

Lysosome Morphology

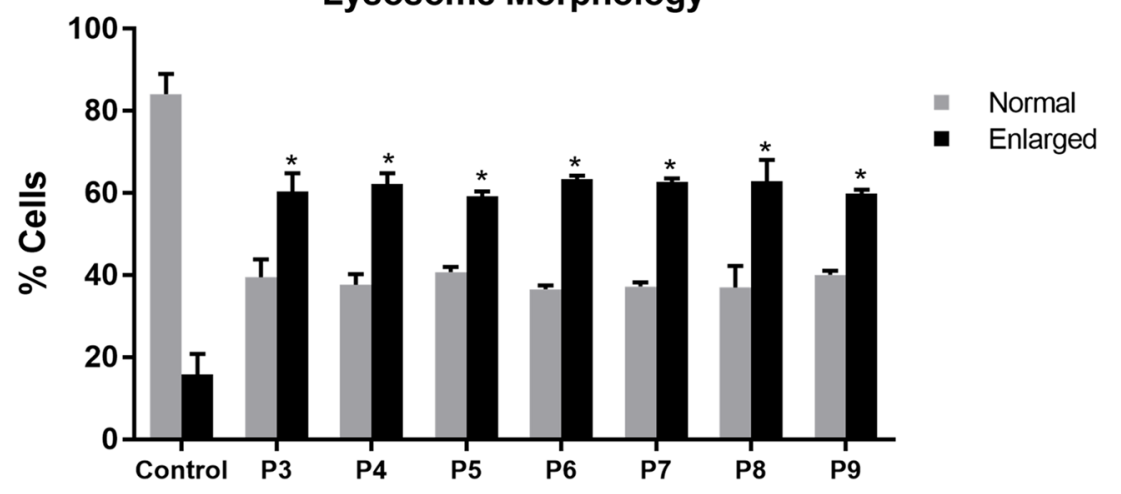

Collectively, our data suggest that various pathogenic variants in MSTOI behave in a similar fashion and lead to mitochondrial abnormalities in patient cells, in particular with regard to mtDNA, providing novel mechanistic insight into the disease pathogenesis associated with MSTO1 mutations.

\section{Discussion}

Bi-allelic pathogenic variants in the nuclear-encoded cytosolic protein $M S T O 1$ have been reported in 12 patients from 7 families to date $[6,16,31,36]$. This study characterizes 12 families with an additional 15 affected patients and thus presents the largest single cohort of patients with variants in the cytosolic mitochondrial fusion regulator, MSTO1. The series includes several novel pathogenic variants and allows for further delineation of the recessive MSTO1-related disease-associated phenotype. We found this phenotype in our cohort to be remarkably consistent with childhood-onset, fairly non-progressive muscle weakness and clinical evidence of corticospinal tract and cerebellar involvement. As a corollary to this clinical presentation, an elevated CK level associated with a histologically dystrophic myopathy and early-onset/congenital yet stable cerebellar atrophy/hypoplasia is seen on testing. Clinical findings are similar to a recently reported small case series of 12 patients with recessive MSTO1-related disease [6, 16, 23, 31, 35]. Pigmentary retinopathy was previously observed as part of the recessive phenotype [23,35]. This finding was not reported in any of the patients in our cohort; however, formal ophthalmologic examination was not pursued in all patients. Arthrogryposis was also previously reported in one patient [23]. While congenital onset hypotonia was noted in three of our patients, no other abnormalities were reported at birth.

The delayed motor development seen in our patients could be due to the cerebellum volume loss, the muscular 
a

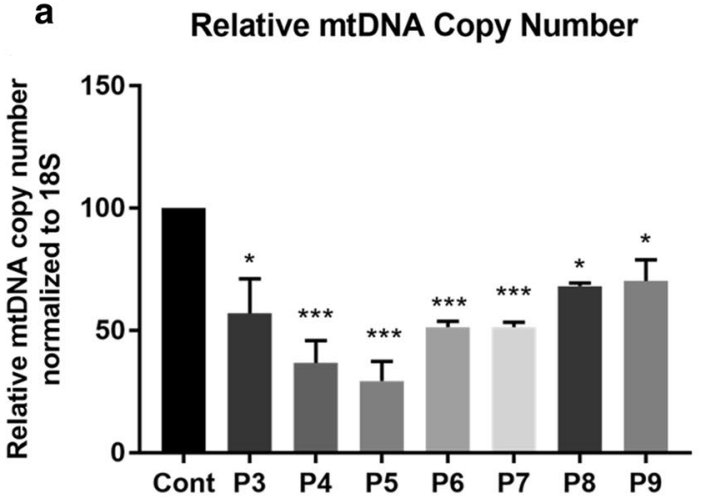

C mtDNA Nucleoid Size Distribution

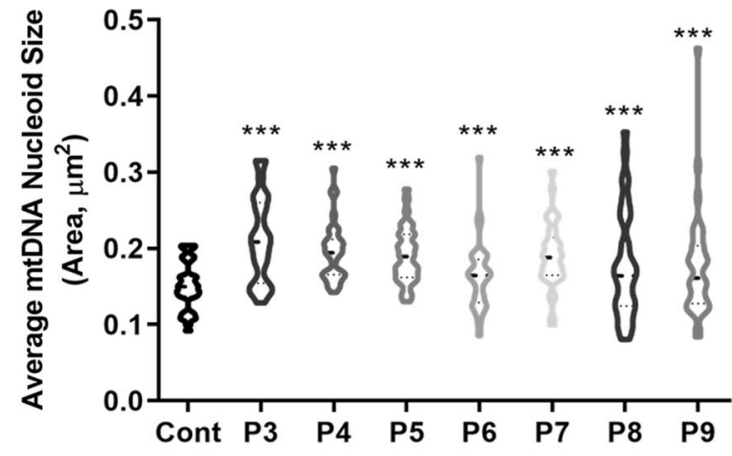

Fig. 6 Pathogenic variants in MSTO1 are linked to mtDNA depletion. a Relative mtDNA copy number normalized to the nuclear-encoded $18 \mathrm{~S}$ gene. Data represent at least three independent biological replicates. b Analysis of mtDNA nucleoid counts per cell from 35 cells for each group. c Quantification of nucleoid sizes in control and patient cells. Data represent average nucleoid sizes from the same cells as in

dystrophy or more likely a combination of both. Review of brain MRI imaging in our cohort demonstrated that the decrease in cerebellar volume is evident in both hemispheres; the volume loss in the vermis is present at a very young age (as observed on the first MRIs obtained) and is non-progressive on follow-up imaging in the majority of patients. Hence, it is possible that the cerebellar volume loss reflects more of a hypoplasia rather than an early-onset progressive atrophy, or a combination thereof. In early cerebellar hypoplasias that interfere with normal cerebellar development, granule cell proliferation deficiency as well as some granular cell loss may underlie an overall smaller cerebellar cortical volume [19]. This may be in keeping with the slight progression of cerebellum volume loss seen in consecutive scans obtained for P12 (p.(Arg345His); p.(Thr324Ile)) at ages 1 and 2 years, which may be disproportionate to the overall brain volume and reflective of a developmental process given that cerebellum growth continues after birth [1]. Without further morphological autopsy data, the relative contributions of hypoplasia versus atrophy will have to remain undetermined.

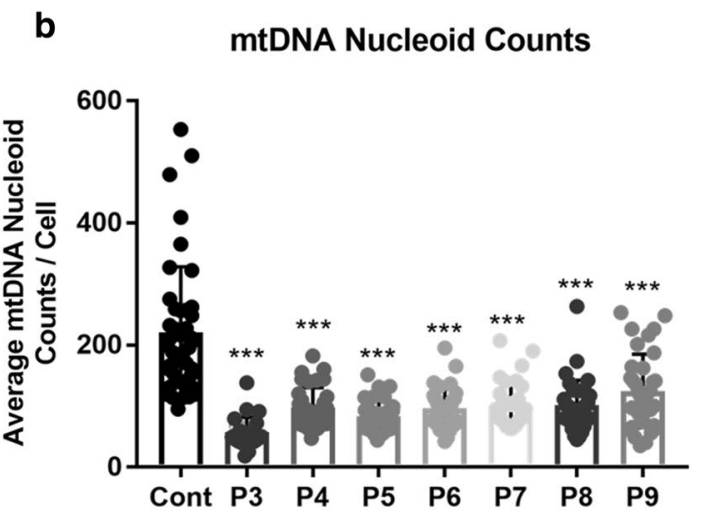

d Frequency of large mtDNA nucleoids

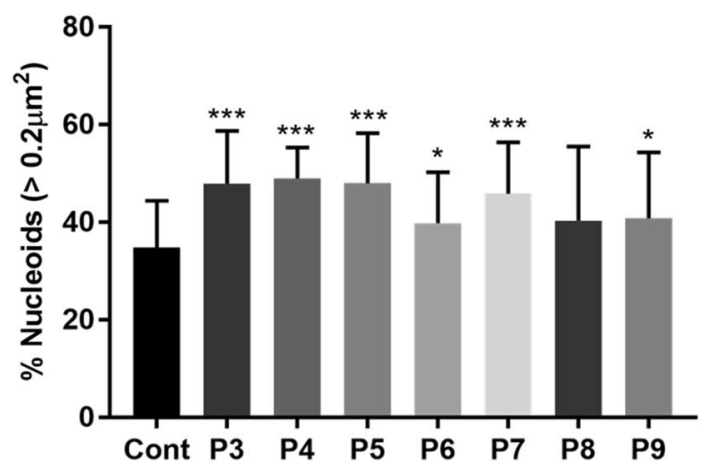

b. Average mtDNA nucleoid size is presented in a violin plot. $\mathrm{K}-\mathrm{S}$ test was performed to determine statistical significance. d Frequency of nucleoids larger than $0.2 \mu^{2}$ in all 35 cells quantified per fibroblast line. Student $T$ test was performed as indicated for $\mathbf{a}, \mathbf{c}$ and $\mathbf{d}$. $* p<0.05, * * p<0.01, * * * p<0.0001$

Clinically, it is reassuring that all patients achieved ambulation, which has been maintained at age 52 years in the oldest patient reported to date with MSTO1-related disease [P14; p.(Phe217Leu); p.(Arg279Cys)].

One of the most important diagnostic considerations in patients presenting with childhood-onset muscle weakness, elevated CK and structural brain abnormalities with prominent cerebellar involvement, includes the $\alpha$-dystroglycanopathies ( $\alpha \mathrm{DGs}$ ). The $\alpha \mathrm{DGs}$ are a clinical and genetic heterogenous sub-group within the congenital muscular dystrophies (CMDs) that manifest as an earlyonset dystrophic muscle disease with central nervous system involvement, including abnormal neuronal migration resulting in cortical malformations as well as impaired synaptic function [17, 37]. Specifically, $\alpha$ DGs caused by mutations in $I S P D$ and GMPPB may manifest with phenotypes reminiscent of recessive $M S T O 1$-related disease [9, $11,17]$. However, hypoglycosylation of $\alpha$-dystroglycan is a distinctive marker for the $\alpha \mathrm{DGs}$ that can be detected using specific antibodies against the matriglycan glycoepitope 
a MST01-P2A-mCherry PicoGreen MitoTracker Deep Red
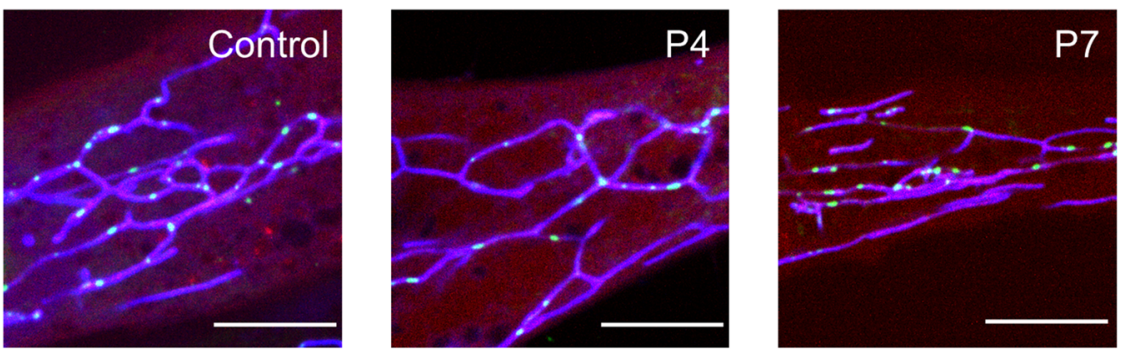

b MSTO1-P2A-mCherry TOMM20 LAMP1
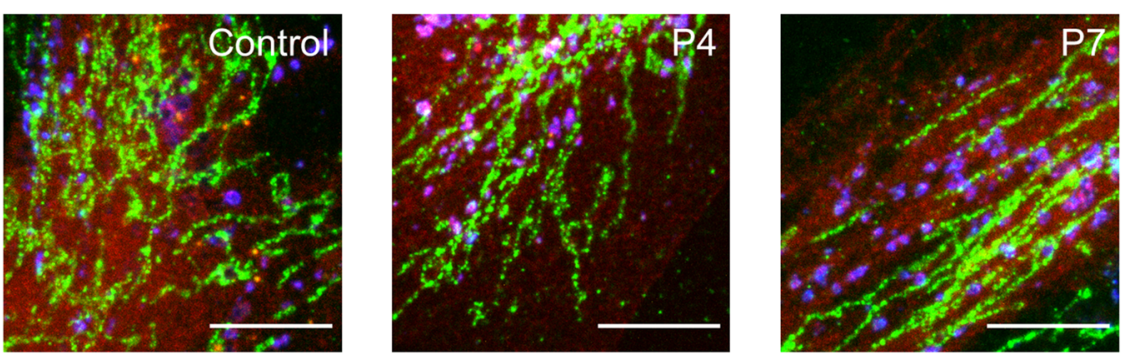

C

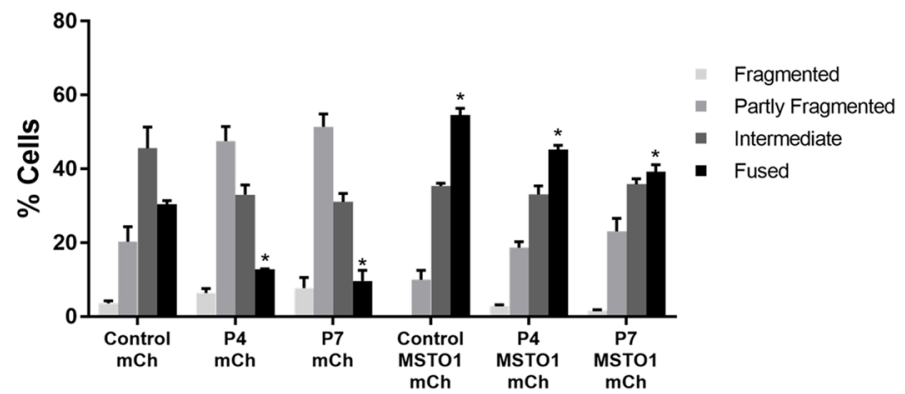

d

Lysosome Morphology

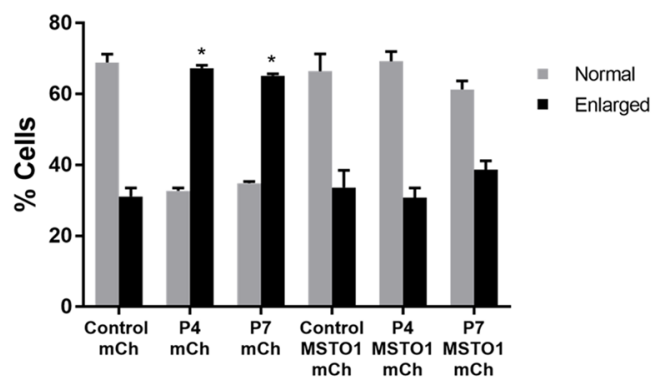

g

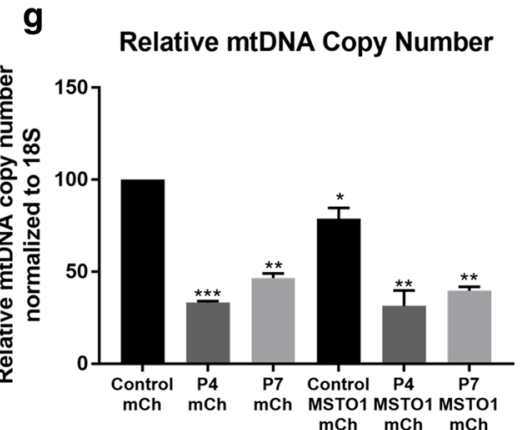

Fig. 7 Expression of wild-type MSTO1 rescues cellular phenotypes in MSTO1 patient fibroblasts. Control, $\mathrm{P} 4$ and $\mathrm{P} 7$ fibroblast cells were transfected with MSTO1-P2A-mCherry or the mCherry empty vector control. Representative images of fibroblasts transfected with MSTO1-P2A-mCherry, for a live cells stained with picogreen and MitoTracker Deep Red, or $\mathbf{b}$ fixed cells stained with antibodies against TOMM20 (green, mitochondria) and LAMP1 (blue, lysosomes). Scalebars: $10 \mu \mathrm{m}$. Transfected fibroblasts, as identified by cytosolic mCherry signal, were characterized as described above for the following cellular phenotypes: c mitochondrial morphology, d lysosome morphology, e average mtDNA nucleoid size, f mtDNA nucleoid counts, and $\mathbf{g}$ relative mtDNA copy number. Student $T$ test was performed as indicated for $\mathbf{c}, \mathbf{d}, \mathbf{f}$, and $\mathbf{g}$. K-S test was performed to determine statistical significance for e. $* p<0.05$, $* * p<0.01$, $* * * p<0.0001$ 
of $\alpha$-dystroglycan on muscle immunohistochemistry and western blot, which would be normal in MSTO1-related disease. In fact, immunofluorescence analysis of the muscle biopsy from P8 (p.(Phe217Leu); p,(Asp236His) and P9 (p.(Tyr478Cys); missing) showed normal $\alpha$-dystroglycan glycoepitope staining (data not shown). The other highly relevant differential diagnosis with a reminiscent clinical spectrum is Marinesco-Sjogren syndrome (MSS) caused by bi-allelic mutations in SIL1. MSS is characterized by intellectual disability, early onset cataracts, ataxia with cerebellar atrophy and myopathy. The absence of cataracts and severe intellectual disability appear to distinguish MSTO1-related disorders from MSS. Approximately $60 \%$ of patients with the classic clinical features of MSS harbour SIL1 pathogenic variants, whereas only $3 \%(1 / 37)$ of those with atypical features have readily identifiable SILI pathogenic variants. Notably, in the study of SILI negative, atypical MSS patients, one patient was ultimately diagnosed with an $A G K$ related mtDNA depletion syndrome [26]. Therefore, it is possible that other atypical MSS patients may also harbour pathogenic MSTO1 variants. In order to help facilitate an accurate genetic diagnosis, MSTOI should be included in targeted next-generation-based neuromuscular, mitochondrial and ataxia-related panels, where MSTOI is currently not included.

In this cohort we report six novel MSTO1 missense variants, a single base pair deletion and a large genomic deletion. Variants are scattered throughout the MSTOI gene and do not preferentially impact specific domains. We identified apparent recurring variants in three specific residues (p.(Phe217Leu), p.(Arg279His) and p.(Asp236His/Gly)). The p.(Arg279His) variant was previously reported as pathogenic in three families in heterozygosity with a truncating allele [23, 31]. There is one individual listed in ExAC who is homozygous for the p.(Arg279His) variant, which would be unusual for a childhood-onset disease [29]. It is, therefore, likely that in its own right this may be a much milder pathogenic allele that needs to occur in compound heterozygosity with a more severe allele in order to manifest as early-onset MSTO1-deficiency [23]. As noted, all patients thus presented with a remarkably homogeneous phenotype; therefore, no clear genotype-phenotype correlations emerged for recessive $\mathrm{MSTO1}$ variants beyond the p.(Arg279His) observation, and the absence of bi-allelic null mutations.

The MSTOI gene is part of a large tandem segmental duplication of approximately $240 \mathrm{~kb}$ located on chromosome 1q22. This arrangement is the result of an evolutionary duplication event estimated to have occurred 37 million years ago in the human evolutionary lineage [28], which also resulted in the derivation of the pseudogene MSTO2P (NR_024117). This locus generates a long non-coding RNA with a nucleotide identity degree of $99.5 \%$ and $98.1 \%$ of exonic and intronic regions, respectively [28]. This high sequence similarity results in ambiguity of alignment of the short-read sequences typically generated with next-generation based genetic testing approaches, as the alignment of short reads to their proper genomic location maps equally well with both MSTO1 and MSTO2P. Consequently, the coverage of MSTO1 is significantly reduced in WES, WGS and RNA-seq data from our patients, resulting in diagnostic challenges. In fact, even with targeted Sanger sequencing it can be challenging to unambiguously sequence MSTOI without contribution of the pseudogene sequence. In this context we also report the first presumed multi-exon deletion of MSTO1, which, as expected, was not identified through WES. In this patient [P7; (p.(Leu450Phe); deletion)], WES identified an apparently homozygous MSTO1 missense variant in the absence of consanguinity; however, subsequent WGS and RNA sequencing failed to identify the deletion due to the inherent difficulties of mapping highly homologous regions. Targeted array CGH analysis with exon-level resolution was able to identify the exon 9-14 deletion. Given the duplicated and thus highly similar regions in this analysis, there were only three probes discriminating MSTOI from $M S T O 2 P$; therefore, the mapping of the deletions to MSTO1 is still ambiguous. Given the diagnostic confidence in the disease phenotype, the reduced MSTO1 protein in this patient's fibroblasts and the previously identified rare, predicted to be damaging missense MSTOI variant, we suspect that the deletion is likely encompassing MSTO1. In contrast, in patient P9 (p.(Tyr478Cys); missing), who also presented with the disease phenotype and reduced MSTO1 protein levels, extensive next-generation based-sequencing including array CGH analysis only yielded a single heterozygous rare, predicted to be damaging missense $\mathrm{MSTOI}$ variant. A pathogenic variant on the other allele was not readily detectable with available technology, suggesting a more complex genomic re-arrangement which may be copy number neutral. Validation work is in progress; however, this work is significantly complicated by the high sequence similarity between the duplicated genomic regions of the locus. Thus proper diagnosis of MSTO1-deficiency may require specialized sequencing strategies, triggered by proper phenotypic recognition through detailed clinical examination, brain MRI and if needed MSTO1 protein analysis in fibroblasts [15].

In all seven novel MSTO1 fibroblast lines characterized, MSTO1 protein was reduced (Fig. 3a), and mitochondrial network fragmentation was observed (Fig. 4). Consistent with our findings, it has been shown previously that the levels of the mitochondrial fusion proteins MFN1/2 and OPA1 are not affected in MSTOI patient cells (Fig. 3b), [16], suggesting that the observed fragmented mitochondrial network phenotype in patient cells is related to MSTO1-deficiency. While defects in mitochondrial fusion have been linked 
to abnormalities in mtDNA, the specific consequences of pathogenic $M S T O 1$ variants regarding mtDNA integrity have not been thoroughly investigated thus far [47]. Of the previous MSTO1 studies, three did not investigate mtDNA [16, 31]. Meanwhile, Nasca et al. reported mtDNA depletion in muscle from patient $\mathrm{A} 1$ and fibroblasts from patients A1 and A2 (sisters), while mtDNA nucleoid size was not quantified [36]. Analysis of mtDNA copy number across our patient cohort now provides further insights into the role of impaired mitochondrial fusion and mtDNA depletion in MSTO1-deficient patient cells. It would be of interest to study this phenomenon in muscle tissue from MSTO1deficient patients, which unfortunately were not available in this cohort.

While the mechanistic link between mitochondrial fusion/ fission dynamics and loss of mtDNA content remains unresolved, the present study adds another mitochondrial fusion protein, MSTO1 to the list of mitochondrial dynamics proteins that are implicated in the maintenance of mtDNA content. Our observation of enlarged nucleoids using PicoGreen staining is of interest in this context. The enlarged nucleoids could reflect changes in the topology of mtDNA that affect the ability of the dye to intercalate [20]. Alternatively, impaired segregation of mtDNA nucleoids, which typically contain only a single copy of the mtDNA genome [27], could lead to multiple mtDNA molecules within close spatial proximity that appear as larger nucleoids [8]. We favour the latter hypothesis given that loss of fusion is known to impair nucleoid distribution with some mitochondrial fragments containing a relatively higher concentration of mtDNA molecules while others completely lack mtDNA [10], which we also observe in MSTO1 fibroblasts (Fig. 4a). Meanwhile, recent work suggesting that reduced fusion causes mtDNA depletion due to insufficient distribution of the mtDNA replication machinery [44] is also consistent with our findings in MSTOI fibroblasts. In particular, the fact that we see a full recovery of mitochondrial and lysosome morphology at $48 \mathrm{~h}$, but only a partial recovery of mtDNA (in the form of restoration of nucleoid size), further suggests that impairments in mitochondrial dynamics are upstream of mtDNA depletion in MSTO1 patient fibroblasts. Nonetheless, the exact link between mtDNA copy number and enlarged nucleoids remains unresolved.

It is also notable that mitochondrial fission was recently shown to be important for mtDNA nucleoid segregation following mtDNA replication [30] and that impairments in fission are known to lead to enlarged nucleoid structures [8]. Taken together, these observations provide further evidence that mitochondrial fusion and fission are important for mtDNA segregation and distribution. We posit that altered distribution and segregation of mtDNA (i.e. nucleoid clumping), in conjunction with reduced mitochondrial fusion, impairs mtDNA maintenance leading to mtDNA depletion.
Importantly, the degree of mtDNA depletion we see in MSTO1 patient fibroblasts (30-70\% of the normal content) is consistent with the levels of mtDNA depletion reported in fibroblasts from OPA1 or MFN2 patients [41, 46, 49] as well as in mtDNA depletion syndromes (Fig. 6) [38]. The clinical significance of this observation requires further studies as the remarkably consistent and homogenous phenotype of recessive $M S T O 1$-related disease reported within our cohort is in contrast to the frequently highly variable clinical spectrum observed in mitochondrial diseases with childhood onset due to either nuclear DNA or mtDNA pathogenic variants. Thus, the consequences of abnormal mitochondrial dynamics caused by recessive MSTO1 pathogenic variants may be less susceptible to acutely variable changes in metabolic demands, while likely incorporating a developmental component given the early clinical manifestations and neuroimaging abnormalities. This consistent phenotype of MSTO1-deficiency reported to date also seems in contrast to the single family with a dominantly acting MSTO1 variant in whom psychiatric manifestations including schizophrenia and autism were leading clinical features, while muscle weakness was mild and cerebellar involvement was not reported [16], thus suggesting a different underlying pathogenic mechanism. It is notable that the pathogenic recessive variants in MSTOI investigated to date seem to impair protein stability in fibroblasts, which is consistent with a loss of function mechanism, while not providing further insight into functional subdomains of the protein. Since haploinsufficiency, as is present in some of the heterozygous parents in our cohort, does not cause a clinical phenotype, the mechanism for the dominantly acting heterozygous mutation may be dominant-negative in nature with perhaps different physiological consequences [16].

Despite evidence for the role of MSTO1 in mitochondrial fusion, the molecular structure of the MSTO1 protein and the exact mechanism by which MSTO1 performs this function directly or via mediators are still unknown. Our findings that bi-allelic loss-of-function variants in MSTO1 result in fragmented mitochondrial networks with mtDNA depletion and nucleoid abnormalities highlight a previously unappreciated role for MSTO1 in the maintenance of the mitochondrial genome. Taken together, our findings newly characterize MSTO1-deficiency as a syndrome of both impaired mitochondrial fusion as well as of mtDNA depletion, while clinically manifesting with a remarkably consistent phenotype.

Acknowledgements We would like to thank the families for their participation, Dr. Sandra Cooper for her expertise along with Christopher Mendoza, Gilberto Averion, Mary Anderson and Linda MacLaren for their help in clinic. We would like to thank the NIH Intramural Sequencing staff for their help with the exome analysis and the Exome Aggregation Consortium and the groups that provided exome variant 
data for comparison. A full list of contributing groups can be found at http://exac.broadinstitute.org/about.

Funding The work in C.G. Bönnemann's laboratory is supported by intramural funds from the NIH National Institute of Neurological Disorders and Stroke. The work in T.E. Shutt's laboratory is supported by funds provided by the Alberta Children's Hospital Research Institute, the Alberta Children's Hospital Foundation, and National Sciences and Engineering Research Council of Canada (NSERC). Exome sequencing was partially funded through the Clinical Center Genomics Opportunity, which is sponsored by the National Human Genome Research Institute, the NIH Deputy Director for Intramural Research and the NIH Clinical Center. Sequencing analysis was provided by the Broad Center for Mendelian Genomics (UM1 HG008900), which is funded by the National Human Genome Research Institute with supplemental funding provided by the National Heart, Lung and Blood Institute under the Trans-Omics for Precision Medicine (TOPMed) program and the National Eye Institute. Part of this work was also performed under the Care4Rare Canada Consortium funded by Genome Canada and the Ontario Genomics Institute (OGI-147), the Canadian Institutes of Health Research, Ontario Research Fund, Genome Alberta, Genome British Columbia, Genome Quebec and Children's Hospital of Eastern Ontario Foundation. Dr. Moore is partially supported by NIH funding of the Iowa Wellstone Muscular Dystrophy Cooperative Research Center, U54, NS053672. R.S. is a recipient of a Queen Elizabeth II Graduate Scholarship and Alberta Children's Hospital Research Institute Graduate Studentship.

\section{Compliance with ethical standards}

Conflict of interest RES is an employee of GeneDx, Inc., a wholly owned subsidiary of OPKO Health, Inc. The other authors report no competing interests.

Open Access This article is distributed under the terms of the Creative Commons Attribution 4.0 International License (http://creativeco mmons.org/licenses/by/4.0/), which permits unrestricted use, distribution, and reproduction in any medium, provided you give appropriate credit to the original author(s) and the source, provide a link to the Creative Commons license, and indicate if changes were made.

\section{References}

1. Adle-Biassette H, Golden JA, Harding B (2017) Developmental and perinatal brain diseases. Handbook Clin Neurol 145:51-78. https://doi.org/10.1016/B978-0-12-802395-2.00006-7

2. Amati-Bonneau P, Guichet A, Olichon A, Chevrollier A, Viala $\mathrm{F}$, Miot $\mathrm{S}$ et al (2005) OPA1 R445H mutation in optic atrophy associated with sensorineural deafness. Ann Neurol 58:958-963. https://doi.org/10.1002/ana.20681

3. Amati-Bonneau P, Valentino ML, Reynier P, Gallardo ME, Bornstein B, Boissiere A et al (2008) OPA1 mutations induce mitochondrial DNA instability and optic atrophy 'plus' phenotypes. Brain 131:338-351. https://doi.org/10.1093/brain/awm298

4. Amberger JS, Bocchini CA, Schiettecatte F, Scott AF, Hamosh A (2015) OMIM.org: online Mendelian Inheritance in Man (OMIM(R)), an online catalog of human genes and genetic disorders. Nucleic Acids Res 43:D789-D798. https://doi.org/10.1093/ nar/gku1205

5. Anderson S, Bankier AT, Barrell BG, de Bruijn MH, Coulson AR, Drouin J et al (1981) Sequence and organization of the human mitochondrial genome. Nature 290:457-465
6. Ardicli D, Sarkozy A, Zaharieva I, Deshpande C, Bodi I, Siddiqui A et al (2019) A novel case of MSTO1 gene related congenital muscular dystrophy with progressive neurological involvement. NMD, Neuromuscular disorders. https://doi.org/10.1016/j. nmd.2019.03.011

7. Ashley N, Harris D, Poulton J (2005) Detection of mitochondrial DNA depletion in living human cells using PicoGreen staining. Exp Cell Res 303:432-446. https://doi.org/10.1016/j.yexcr .2004.10.013

8. Ban-Ishihara R, Ishihara T, Sasaki N, Mihara K, Ishihara N (2013) Dynamics of nucleoid structure regulated by mitochondrial fission contributes to cristae reformation and release of cytochrome c. Proc Natl Acad Sci USA 110:11863-11868. https://doi. org/10.1073/pnas.1301951110

9. Carss KJ, Stevens E, Foley AR, Cirak S, Riemersma M, Torelli $S$ et al (2013) Mutations in GDP-mannose pyrophosphorylase B cause congenital and limb-girdle muscular dystrophies associated with hypoglycosylation of alpha-dystroglycan. Am J Hum Genet 93:29-41. https://doi.org/10.1016/j.ajhg.2013.05.009

10. Chen H, McCaffery JM, Chan DC (2007) Mitochondrial fusion protects against neurodegeneration in the cerebellum. Cell 130:548-562. https://doi.org/10.1016/j.cell.2007.06.026

11. Cirak S, Foley AR, Herrmann R, Willer T, Yau S, Stevens E et al (2013) ISPD gene mutations are a common cause of congenital and limb-girdle muscular dystrophies. Brain J Neurol 136:269281. https://doi.org/10.1093/brain/aws312

12. Demers-Lamarche J, Guillebaud G, Tlili M, Todkar K, Belanger N, Grondin M et al (2016) Loss of mitochondrial function impairs lysosomes. J Biol Chem 291:10263-10276. https://doi. org/10.1074/jbc.M115.695825

13. Eaton JS, Lin ZP, Sartorelli AC, Bonawitz ND, Shadel GS (2007) Ataxia-telangiectasia mutated kinase regulates ribonucleotide reductase and mitochondrial homeostasis. J Clin Invest 117:27232734. https://doi.org/10.1172/JCI31604

14. El-Hattab AW, Scaglia F (2013) Mitochondrial DNA depletion syndromes: review and updates of genetic basis, manifestations, and therapeutic options. Neurother J Am Soc Exp Neuro Ther 10:186-198. https://doi.org/10.1007/s13311-013-0177-6

15. Foley ARD, Bonnemann CG (2015) Next-generation sequencing still needs our generation's clinicians. Next-generation sequencing still needs our generation's clinicians 1:2376-7839

16. Gal A, Balicza P, Weaver D, Naghdi S, Joseph SK, Varnai P et al (2017) MSTO1 is a cytoplasmic pro-mitochondrial fusion protein, whose mutation induces myopathy and ataxia in humans. EMBO Mol Med 9:967-984. https://doi.org/10.15252/emmm.201607058

17. Godfrey C, Foley AR, Clement E, Muntoni F (2011) Dystroglycanopathies: coming into focus. Curr Opin Genet Dev 21:278285. https://doi.org/10.1016/j.gde.2011.02.001

18. Gustafsson CM, Falkenberg M, Larsson NG (2016) Maintenance and expression of mammalian mitochondrial DNA. Annu Rev Biochem 85:133-160. https://doi.org/10.1146/annurev-bioch em-060815-014402

19. Harding BN, Copp AJ (2008) Malformations. In: Love SLD, Ellison DW (eds) Greenfield's neuropathology, 8th edn. CRC Press, Hodder Arnold, London, p 365

20. He J, Mao CC, Reyes A, Sembongi H, Di Re M, Granycome C, Clippingdale AB et al (2007) The AAA+ protein ATAD3 has displacement loop binding properties and is involved in mitochondrial nucleoid organization. J Cell Biol 176:141-146. https://doi. org/10.1083/jcb.200609158

21. Hermann GJ, Thatcher JW, Mills JP, Hales KG, Fuller MT, Nunnari J, Shaw JM (1998) Mitochondrial fusion in yeast requires the transmembrane GTPase Fzo1p. J Cell Biol 143:359-373

22. Hudson G, Amati-Bonneau P, Blakely EL, Stewart JD, He L, Schaefer AM et al (2008) Mutation of OPA1 causes dominant optic atrophy with external ophthalmoplegia, ataxia, 
deafness and multiple mitochondrial DNA deletions: a novel disorder of mtDNA maintenance. Brain 131:329-337. https://doi. org/10.1093/brain/awm272

23. Iwama K, Takaori T, Fukushima A, Tohyama J, Ishiyama A, Ohba C et al (2018) Novel recessive mutations in MSTO1 cause cerebellar atrophy with pigmentary retinopathy. J Hum Genet 63:263-270. https://doi.org/10.1038/s10038-017-0405-8

24. Kelly RD, Mahmud A, McKenzie M, Trounce IA, St John JC (2012) Mitochondrial DNA copy number is regulated in a tissue specific manner by DNA methylation of the nuclear-encoded DNA polymerase gamma A. Nucleic Acids Res 40:10124-10138. https://doi.org/10.1093/nar/gks770

25. Kim JY, Hwang JM, Ko HS, Seong MW, Park BJ, Park SS (2005) Mitochondrial DNA content is decreased in autosomal dominant optic atrophy. Neurology 64:966-972. https://doi.org/10.1212/01. wnl.0000157282.76715.b1

26. Krieger M, Roos A, Stendel C, Claeys KG, Sonmez FM, Baudis $M$ et al (2013) SIL1 mutations and clinical spectrum in patients with Marinesco-Sjogren syndrome. Brain 136:3634-3644. https ://doi.org/10.1093/brain/awt283

27. Kukat C, Davies KM, Wurm CA, Spahr H, Bonekamp NA, Kuhl I et al (2015) Cross-strand binding of TFAM to a single mtDNA molecule forms the mitochondrial nucleoid. Proc Natl Acad Sci USA 112:11288-11293. https://doi.org/10.1073/pnas.15121 31112

28. Kuryshev VY, Vorobyov E, Zink D, Schmitz J, Rozhdestvensky TS, Munstermann E et al (2006) An anthropoid-specific segmental duplication on human chromosome 1q22. Genomics 88:143-151. https://doi.org/10.1016/j.ygeno.2006.02.002

29. Lek M, Karczewski KJ, Minikel EV, Samocha KE, Banks E, Fennell $\mathrm{T}$ et al (2016) Analysis of protein-coding genetic variation in 60,706 humans. Nature 536:285-291. https://doi.org/10.1038/ nature 19057

30. Lewis SC, Uchiyama LF, Nunnari J (2016) ER-mitochondria contacts couple mtDNA synthesis with mitochondrial division in human cells. Science (New York, NY) 353:aaf5549. https://doi. org/10.1126/science.aaf5549

31. Li K, Jin R, Wu X (2019) Whole-exome sequencing identifies rare compound heterozygous mutations in the MSTO1 gene associated with cerebellar ataxia and myopathy. Eur J Med Genet. https://doi. org/10.1016/j.ejmg.2019.01.013

32. Livak KJ, Schmittgen TD (2001) Analysis of relative gene expression data using real-time quantitative PCR and the 2(-Delta Delta C(T)) Method. Methods 25:402-408. https://doi.org/10.1006/ meth.2001.1262

33. Moraes CT, Shanske S, Tritschler HJ, Aprille JR, Andreetta F, Bonilla E (1991) mtDNA depletion with variable tissue expression: a novel genetic abnormality in mitochondrial diseases. Am J Hum Genet 48:492-501

34. Nasca A, Legati A, Baruffini E, Nolli C, Moroni I (2016) Biallelic mutations in DNM1L are associated with a slowly progressive infantile encephalopathy. Hum Mutat 37:898-903. https://doi. org/10.1002/humu.23033

35. Nasca A, Scotton C, Zaharieva I, Neri M, Selvatici R, Magnusson OT et al (2017) Recessive mutations in MSTO1 cause mitochondrial dynamics impairment, leading to myopathy and ataxia. Hum Mutat 38:970-977. https://doi.org/10.1002/humu.23262

36. Nasca A, Scotton C, Zaharieva I, Neri M, Selvatici R, Magnusson OT (2017) Recessive mutations in MSTO1 cause mitochondrial dynamics impairment, leading to myopathy and ataxia. Hum Mutat 38:970-977. https://doi.org/10.1002/humu.23262

37. Nickolls AR, Bonnemann CG (2018) The roles of dystroglycan in the nervous system: insights from animal models of muscular dystrophy. Dis Model Mech. https://doi.org/10.1242/dmm.03593 1

38. Nogueira C, Almeida LS, Nesti C, Pezzini I, Videira A, Vilarinho L (2014) Syndromes associated with mitochondrial DNA depletion. Ital J Pediatr 40:34. https://doi.org/10.1186/1824-7288-40-34

39. Nunnari J, Suomalainen A (2012) Mitochondria: in sickness and in health. Cell 148:1145-1159. https://doi.org/10.1016/j. cell.2012.02.035

40. Potorac I, Rivero-Muller A, Trehan A, Kielbus M, Jozwiak K, Pralong F et al (2016) A vital region for human glycoprotein hormone trafficking revealed by an LHB mutation. J Endocrinol 231:197-207. https://doi.org/10.1530/JOE-16-0384

41. Renaldo F, Amati-Bonneau P, Slama A, Romana C, Forin V, Doummar D et al (2012) MFN2, a new gene responsible for mitochondrial DNA depletion. Brain 135:e223, 221-224. https://doi. org/10.1093/brain/aws111 (author reply e224, 221-223)

42. Sabouny R, Fraunberger E, Geoffrion M, Ng AC, Baird SD, Screaton RA et al (2017) The Keap1-Nrf2 stress response pathway promotes mitochondrial hyperfusion through degradation of the mitochondrial fission protein Drp1. Antioxid Redox Signal 27:1447-1459. https://doi.org/10.1089/ars.2016.6855

43. Schindelin J, Arganda-Carreras I, Frise E, Kaynig V, Longair M, Pietzsch T et al (2012) Fiji: an open-source platform for biological-image analysis. Nat Methods 9:676-682. https://doi. org/10.1038/nmeth.2019

44. Silva Ramos E, Motori E, Bruser C, Kuhl I, Yeroslaviz A, Ruzzenente B et al (2019) Mitochondrial fusion is required for regulation of mitochondrial DNA replication. PLoS Genet 15:e1008085. https://doi.org/10.1371/journal.pgen.1008085

45. Sobreira N, Schiettecatte F, Valle D, Hamosh A (2015) GeneMatcher: a matching tool for connecting investigators with an interest in the same gene. Hum Mutat 36:928-930. https://doi. org/10.1002/humu.22844

46. Spiegel R, Saada A, Flannery PJ, Burte F, Soiferman D, Khayat M et al (2016) Fatal infantile mitochondrial encephalomyopathy, hypertrophic cardiomyopathy and optic atrophy associated with a homozygous OPA1 mutation. J Med Genet 53:127-131. https ://doi.org/10.1136/jmedgenet-2015-103361

47. Suarez-Rivero JM, Villanueva-Paz M, de la Cruz-Ojeda P, de la Mata M, Cotan D, Oropesa-Avila M et al (2016) Mitochondrial dynamics in mitochondrial diseases. Diseases. https://doi. org/10.3390/diseases5010001

48. Suomalainen A, Isohanni P (2010) Mitochondrial DNA depletion syndromes-many genes, common mechanisms. Neuromusc Disorders NMD 20:429-437. https://doi.org/10.1016/j. nmd.2010.03.017

49. Vielhaber S, Debska-Vielhaber G, Peeva V, Schoeler S, Kudin AP, Minin I et al (2013) Mitofusin 2 mutations affect mitochondrial function by mitochondrial DNA depletion. Acta Neuropathol 125:245-256. https://doi.org/10.1007/s00401-012-1036-y

Publisher's Note Springer Nature remains neutral with regard to jurisdictional claims in published maps and institutional affiliations. 


\section{Affiliations}

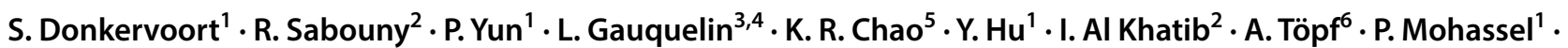
B. B. Cummings ${ }^{5} \cdot$ R. Kaur ${ }^{1}$. D. Saade ${ }^{1}$. S. A. Moore ${ }^{7}$. L. B. Waddell ${ }^{8,9} \cdot$ M. A. Farrar ${ }^{10,11}$. J. K. Goodrich ${ }^{5}$.

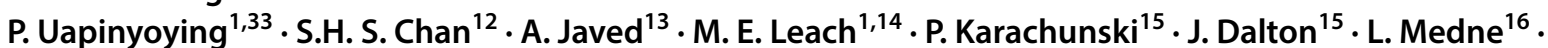
A. Harper ${ }^{17}$. C. Thompson ${ }^{18} \cdot$ I. Thiffault ${ }^{19,20,21}$ - S. Specht ${ }^{6} \cdot$ R. E. Lamont ${ }^{22} \cdot$ C. Saunders ${ }^{19,20,21} \cdot$ H. Racher ${ }^{22}$.

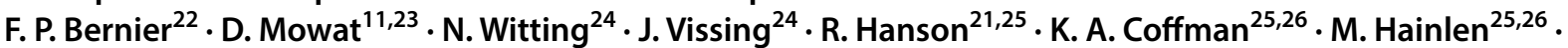
J. S. Parboosingh ${ }^{22} \cdot$ A. Carnevale ${ }^{3}$. G. Yoon ${ }^{3,4} \cdot$ R. E. Schnur ${ }^{27}$. Care4Rare Canada Consortium ${ }^{29} \cdot$ K. M. Boycott ${ }^{28,29}$.

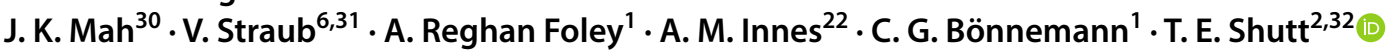

C. G. Bönnemann

Carsten.bonnemann@nih.gov

$\triangle$ T. E. Shutt

timothy.shutt@ucalgary.ca

1 Neuromuscular and Neurogenetic Disorders of Childhood Section, National Institute of Neurological Disorders and Stroke, National Institutes of Health, Bethesda, MD, USA

2 Department of Biochemistry and Molecular Biology, University of Calgary, Calgary, Canada

3 Division of Clinical and Metabolic Genetics, Department of Paediatrics, The Hospital for Sick Children, University of Toronto, Toronto, ON, Canada

4 Division of Neurology, Department of Paediatrics, The Hospital for Sick Children, University of Toronto, Toronto, ON, Canada

5 Center for Mendelian Genomics, Program in Medical and Population Genetics, Broad Institute of MIT and Harvard, Boston, MA, USA

6 John Walton Muscular Dystrophy Research Centre, Institute of Genetic Medicine, Newcastle University, Newcastle upon Tyne, UK

7 Department of Pathology Carver College of Medicine, The University of Iowa, Iowa City, IA, USA

8 Kids Neuroscience Centre, Kids Research, The Children's Hospital at Westmead, Sydney, NSW 2145, Australia

9 Discipline of Child and Adolescent Health, Faculty of Medicine and Health, The University of Sydney, Westmead, NSW 2145, Australia

10 Department of Neurology, Sydney Children's Hospital, Sydney, NSW, Australia

11 UNSW Sydney, School of Women's and Children's Health, Sydney, NSW, Australia

12 Department of Paediatrics and Adolescent Medicine, Queen Mary Hospital, The University of Hong Kong, Hong Kong SAR, China

13 School of Biomedical Science, The University of Hong Kong, Hong Kong SAR, China

14 Oregon Health and Science University, Neuromuscular Program, Doernbecher Children's Hospital, Portland, OR, USA
15 Department of Neurology, University of Minnesota, Minneapolis, MN, USA

16 Division of Human Genetics, The Children's Hospital of Philadelphia, Philadelphia, USA

17 Department of Neurology, Virginia Commonwealth University, Children's Hospital of Richmond at VCU, Richmond, VA, USA

18 Department of Pediatrics, University of California San Diego, San Diego, CA, USA

19 Department of Pathology and Laboratory Medicine, Children's Mercy Hospital, Kansas City, USA

20 Center for Pediatric Genomic Medicine, Children's Mercy Hospital, Kansas City, USA

21 University of Missouri-Kansas City School of Medicine, Kansas City, USA

22 Department of Medical Genetics and Alberta Children's Hospital Research Institute, Cumming School of Medicine, University of Calgary, Calgary, AB, Canada

23 Department of Medical Genetics, Sydney Children's Hospital, Sydney, NSW, Australia

24 Department of Neurology, University Hospital Rigshospitalet, Copenhagen, Denmark

25 Department of Pediatrics, Children's Mercy Hospital, Kansas City, USA

26 Division of Neurology, Children's Mercy Hospital, Kansas City, USA

27 GeneDx, Gaithersburg, MD, USA

28 Children's Hospital of Eastern Ontario Research Institute, University of Ottawa, Ottawa, Canada

29 Care4Rare Research Consortium, Ottawa, Canada

30 Departments of Pediatrics, Section of Neurology, University of Calgary, Calgary, AB, Canada

31 Newcastle Hospitals NHS Foundation Trust, Newcastle upon Tyne, UK

32 Department of Medical Genetics, Alberta Children's Hospital Research Institute, Hotchkiss Brain Institute, University of Calgary, Calgary, Canada

33 Research for Genetic Medicine, Children's National Medical Center, Washington, DC, USA 